\title{
Market-based Coordination of Integrated Electricity and Natural Gas Systems Under Uncertain Supply
}

Ordoudis, Christos; Delikaraoglou, Stefanos; Kazempour, Jalal; Pinson, Pierre

Published in:

European Journal of Operational Research

Link to article, DOI:

10.1016/j.ejor.2020.05.007

Publication date:

2020

Document Version

Early version, also known as pre-print

Link back to DTU Orbit

Citation (APA):

Ordoudis, C., Delikaraoglou, S., Kazempour, J., \& Pinson, P. (2020). Market-based Coordination of Integrated Electricity and Natural Gas Systems Under Uncertain Supply. European Journal of Operational Research, 287(3), 1105-1119. https://doi.org/10.1016/j.ejor.2020.05.007

\section{General rights}

Copyright and moral rights for the publications made accessible in the public portal are retained by the authors and/or other copyright owners and it is a condition of accessing publications that users recognise and abide by the legal requirements associated with these rights.

- Users may download and print one copy of any publication from the public portal for the purpose of private study or research.

- You may not further distribute the material or use it for any profit-making activity or commercial gain

- You may freely distribute the URL identifying the publication in the public portal 


\title{
Market-based Coordination of Integrated Electricity and Natural Gas Systems Under Uncertain Supply
}

\author{
Christos Ordoudis $^{a, *}$, Stefanos Delikaraoglou ${ }^{\mathrm{b}}$, Jalal Kazempour ${ }^{\mathrm{a}}$, Pierre Pinson ${ }^{\mathrm{a}}$ \\ ${ }^{a}$ Department of Electrical Engineering, Technical University of Denmark, Kgs. Lyngby 2800, Denmark \\ ${ }^{b}$ Power Systems Laboratory, ETH, Zurich, Switzerland
}

\begin{abstract}
The interdependence between electricity and natural gas systems has lately increased due to the wide deployment of gas-fired power plants (GFPPs). Moreover, weather-driven renewables introduce uncertainty in the operation of the integrated energy system, increasing the need for operational flexibility. Recently proposed stochastic dispatch models optimally use the available flexibility and minimize the total expected system cost. However, these models are incompatible with the current sequential market design. We propose a novel method to optimally define the available natural gas volume for power production scheduling, anticipating the real-time flexibility needs. This volume-based model is formulated as a stochastic bilevel program that aims to enhance the inter-temporal coordination of scheduling and balancing operations, while remaining compatible with the sequential clearing of day-ahead and real-time markets. The proposed model accounts for the inherent flexibility of the natural gas system via the proper modeling of linepack capabilities and reduces the total expected system cost by the optimal definition of natural gas volume availability for GFPPs at the forward phase. The volume-based coordination model is compared with a price-based coordination alternative, which was recently proposed. In the latter one, the natural gas price perceived by GFPPs is similarly adjusted to enhance the temporal coordination of scheduling and balancing stages. This comparison enables us to highlight the main properties and differences between the two coordination mechanisms.
\end{abstract}

Keywords: OR in energy, bilevel programming, integrated electricity and natural gas systems, market-based coordination, uncertainty.

\section{Introduction}

The coupling between electricity and natural gas systems has been substantially strengthened due to the increased utilization of gas-fired power plants (GFPPs) over the last decades and this trend is expected to continue in the foreseeable future (U.S. Energy Information Administration,

\footnotetext{
${ }^{*}$ Corresponding author

Email addresses: chror@elektro.dtu.dk (Christos Ordoudis), delikaraoglou@eeh.ee.ethz.ch (Stefanos Delikaraoglou), seykaz@elektro.dtu.dk (Jalal Kazempour), ppin@elektro.dtu.dk (Pierre Pinson) 
2016; Meibom et al., 2013; Gil et al., 2014). In addition, renewable energy sources, such as wind and solar power, already comprise a significant share of the generation mix. The co-existence of these two types of power production plants serves as a promising combination for a smooth transition to a sustainable energy system that is flexible enough to accommodate high shares of renewables. To this end, the interdependence between these two energy systems will increase and the intermittency of renewable energy sources will eventually affect the operation of both systems. Hence, there is a compelling need to introduce mechanisms that treat these systems in an integrated manner.

The coordinated operation of electricity and natural gas systems has been extensively studied lately. Zlotnik et al. (2017) indicate the benefits of improved coordination between the two energy systems under high intraday variability of GFPPs' fuel consumption. In a similar context, the impact of natural gas supply uncertainty and price variability on the power system dispatch is analyzed by Zhao et al. (2016), showing that these parameters can considerably alter the market outcomes. Moreover, Correa-Posada \& Sanchez-Martin (2014) highlight the benefit in terms of improved flexibility and reliability, when accounting for the ability to store natural gas in the pipelines, known as linepack. Therefore, both physical and economic links between electricity and natural gas systems have an eminent role in short-term operations and are highly essential in the presence of renewables. He et al. (2017) examine the effect of uncertain renewable power production to the coupled energy system in a robust co-optimization framework, while Alabdulwahab et al. (2015) utilize a model based on stochastic programming to dispatch the power system with feasible fuel supply from the natural gas network. Moreover, Zeinalzadeh et al. (2017) consider a joint optimization framework that utilizes GFPPs to firm up uncertain power supply from renewables.Taking a market perspective, Wang et al. (2018) propose an equilibrium model for the interdependent electricity and natural gas markets that allows for short-term energy trading based on locational marginal prices. In a similar context, this paper explores the short-term coupled operation of electricity and natural gas systems in a market framework where these energy commodities are traded based on their marginal prices. This approach is highly relevant especially when the two systems are operated by the same entity, e.g. Energinet.dk in Denmark, which operates both electricity and natural gas systems (Pinson et al., 2017).

Following the paradigm of the electricity sector, the volume of natural gas traded in the spot markets is continuously increasing (Pinson et al., 2017). Therefore, the short-term operation of electricity and natural gas systems should be modeled following a market-based framework. The short-term operation is mainly associated with two trading floors, namely the day-ahead and balancing markets that are cleared in a sequential manner. The day-ahead market is settled 12-36 hours ahead of the actual system operation, while the balancing market in cleared close to realtime operation to deal with the necessary adjustments to keep the system balanced. However, this sequential arrangement is inefficient under high shares of stochastic renewable power production due to its deterministic view of the uncertain renewables' production. Stochastic programming has been utilized to enhance the temporal coordination between these two trading floors by employing 
a probabilistic description of stochastic renewable production in an electricity-only market design (Morales et al., 2012; Pritchard et al., 2010) but also in an integrated electricity and natural gas market framework (Alabdulwahab et al., 2015; Ordoudis et al., 2019b). More specifically, in (Ordoudis et al., 2019b), we proposed a coupled electricity and natural gas scheduling approach based on two-stage stochastic programming that efficiently accounts for the dynamics of the natural gas flow. In this work, two key coordination parameters; namely, the natural gas price and volume availability for power production from GFPPs, were identified to have an impact on the dispatch of the coupled energy system.

Despite that the stochastic dispatch models provide the solution with the minimum expected system cost, they are incompatible with the current market design, while they also suffer from some design flaws related to the violation of the least-cost merit-order principle ${ }^{1}$ as shown by Morales et al. (2014) and Zavala et al. (2017). For this reason, several approaches have been proposed that aim at approximating the stochastic ideal solution, while maintaining the current market architecture regarding the temporal sequence of the day-ahead and real-time trading floors. Exploiting the natural gas price coordination parameter identified by Ordoudis et al. (2019b), we presented a price-based coordination model in (Ordoudis et al., 2017). In particular, a systematic approach to generate proper flexibility price signals that adjust the natural gas price perceived by GFPPs was proposed in order to provide a market outcome that is closer the stochastic ideal solution. In a similar vein, Morales et al. (2014) proposed an improved dispatch model that minimizes expected system's cost and respects the merit-order principle by scheduling wind power in a value different than its expected production, while cost recovery ${ }^{2}$ of flexible producers is guaranteed for any realization of uncertainty. Additionally, Jensen et al. (2018) developed a framework to set the available transfer capacity (ATC) among zones in a cost-optimal manner and attain a solution closer to the stochastic one, while Dvorkin et al. (2019) approximated the stochastic solution by optimally setting the reserve requirements. Finally, Delikaraoglou \& Pinson (2019) proposed a model that efficiently dispatches the power system with an optimal setting of allocation between energy and reserves on the inter-regional HVDC interconnections. In the aforementioned works, the system operator is able to properly tune these parameters, i.e. natural gas price, day-ahead wind power schedule, ATCs, HVDC allocation, reserve requirements, in order to communicate the missing information to the day-ahead stage and improve its temporal coordination with the balancing stage.

While the end-goal of this work remains the improvement of temporal coordination between the scheduling and dispatch operations of the integrated electricity and natural gas system, we propose a new coordination mechanism that aims to exploit the volume link between the two

\footnotetext{
${ }^{1}$ This principle defines the ranking of power producers based on an ascending order of their short-run marginal cost and the respective power to be generated. The power producers are thus scheduled to meet the demand in a way that those with the lowest marginal cost are scheduled first, which results in minimizing the system cost.

${ }^{2}$ Revenue of each market participant is greater than or equal to its operating costs.
} 
systems as it was identified by Ordoudis et al. (2019b). To this end, we formulate a systematic method to define the optimal natural gas volume that is made available for power production at the day-ahead stage. This approach has a fundamental difference compared to the price-based coordination structure proposed by Ordoudis et al. (2017), since it only adjusts purely technical parameters at the interface of the two systems, without interfering with the economic parameters of the market. This approach resembles the "maximum gas burn" constraint that was recently introduced by the California Independent System Operator (CAISO) to address reliability risks due to the limited operability of the Aliso Canyon natural gas storage facility (CAISO, 2017).

In this volume-based model, we consider the natural gas availability as a parameter that can be controlled by the operator and we build a stochastic bilevel model to determine its optimal value, while anticipating the future balancing needs due to forecast errors from uncertain power supply. This mechanism aims to improve the temporal coordination between day-ahead and balancing trading floors by preserving the existing market architecture and approximating the efficiency of the stochastic solution that is obtained by Ordoudis et al. (2019b), under the fundamental assumption of co-optimized scheduling and balancing operations. The natural gas availability only affects the day-ahead schedule of the integrated energy system and the real-time balancing takes into account the physical characteristics of the two networks. The proposed model can be utilized to provide further insights into the economic and technical implications resulting from the improved temporal coordination that is achieved by defining the natural gas volume availability before any clearing procedure. Therefore, we do not advocate for the adoption of this model as an actual market-clearing algorithm, but rather than as a decision-support tool that improves the cost efficiency of the integrated energy system. Regarding the natural gas system modeling, we adopt the approach proposed by Ordoudis et al. (2019b) that permits to model the linepack capability and make optimal use of the available network flexibility. It should be noted that this detailed gas network representation cannot be integrated in the model proposed by Ordoudis et al. (2017), since the resulting mathematical formulation would violate the convexity requirements that are necessary to obtain a tractable reformulation.

The remainder of the paper is organized as follows. Section 2 outlines the main properties of each dispatch model for the integrated energy system, while the mathematical formulation is presented in Section 3. The results are illustrated in Section 4, and Section 5 concludes the paper. The guarantee of cost recovery for flexible producers in the sequential arrangement of day-ahead and balancing stages is further discussed and proven in the appendix of the manuscript. A detailed nomenclature is also provided in the appendix. For the sake of clarity and completeness, the price-based dispatch model proposed by Ordoudis et al. (2017) is discussed and compared with the volume-based one in the present work. Finally, additional material including some mathematical extensions and additional results are given in the online appendix (Ordoudis et al., 2019a). 


\section{Market-based Coordination}

In this paper, we study four dispatch models for scheduling and balancing electricity and natural gas systems. These models achieve different degrees of temporal coordination for the integrated energy system. This section describes their fundamental principles and provides a schematic representation to outline the main properties of each coordination scheme.

\subsection{Sequential Dispatch of Integrated Energy System}

The sequential dispatch of an integrated energy system $(S e q)$ models a case of perfect intersystems coordination between electricity and natural gas networks for both day-ahead and balancing markets, as shown in Fig. 1. However, these market floors are cleared in sequential and independent auctions, resulting to imperfect temporal coordination between the scheduling and balancing operations. Having as input a single-valued forecast of the stochastic power production, a common day-ahead market is cleared to obtain the initial operation schedule for both systems. Getting closer to actual operation, when the realization of stochastic production $\omega^{\prime}$ is known, the balancing actions to compensate for potential forecast errors are jointly optimized for both systems through a common balancing market. Even though this sequential approach may be inefficient due to imperfect temporal coordination, it admits an important economic property that ensures cost recovery for flexible producers for any realization of stochastic production, as shown in the appendix. Assuming a co-optimization process that minimizes the combined system cost at each market stage, this setup deviates from the current design since it does not respect the asynchronous timing and the independent clearing of the respective markets (Hibbard \& Schatzki, 2012). However, this market model allows us to assess the net value of temporal coordination between the day-ahead and balancing markets of interdependent energy networks.

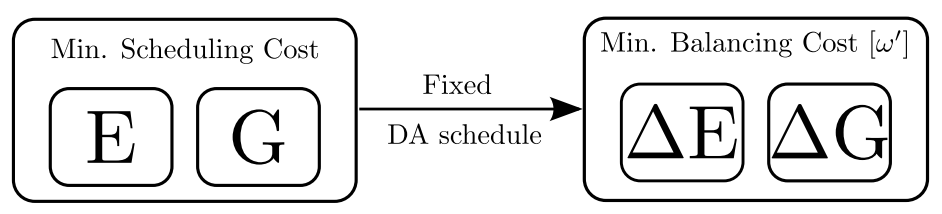

Figure 1: Sequential dispatch of integrated energy system. DA: Day-ahead, E: Electricity, G: Natural gas, $\Delta$ E: Electricity adjustment, $\Delta \mathrm{G}$ : Natural gas adjustment.

\subsection{Stochastic Dispatch of Integrated Energy System}

To improve the temporal coordination between the scheduling and balancing operations, we construct the stochastic coupled electricity and natural gas dispatch model (Stoch) illustrated in Fig. 2 and previously proposed by Ordoudis et al. (2019b). Here, the day-ahead market cooptimizes the electricity and natural gas schedules based on a probabilistic description of uncertain supply, which allows to anticipate the cost of re-dispatch actions in real-time operation. Such probabilistic description is based on the available forecast at the day-ahead stage and may not 
cover the exact realization in real-time. However, given that the uncertainty modelling adequately captures the true characteristics of the stochastic processes involved, the potential realization will be represented via a set of scenarios $\Omega$. This market setup is mathematically formulated as a twostage stochastic programming problem that minimizes the total expected cost of the integrated energy system. The definition of this model provides an ideal reference solution that attains perfect inter-systems and temporal coordination, assuming that a realistic range and probability distribution of scenarios are considered. However, its real-life implementation is restricted because cost recovery for market participants and revenue adequacy ${ }^{3}$ for the system operator hold only in expectation (Morales et al., 2012). Actually, these fundamental economic properties may be violated for some uncertainty realizations in scenario set $\Omega$ since this model does not respect the least-cost merit-order principle in the day-ahead market.

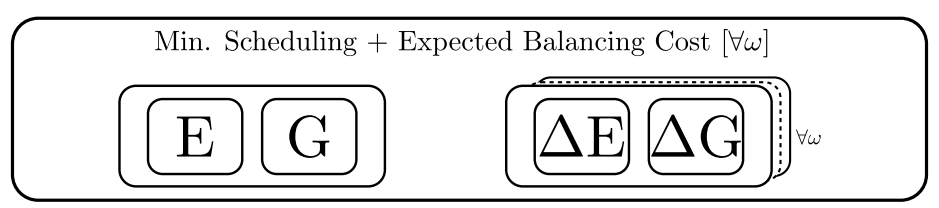

Figure 2: Stochastic dispatch of integrated energy system.

\subsection{Volume-based Coordination in Sequential Dispatch of Integrated Energy System}

Aiming to address the caveat of imperfect temporal coordination of the Seq model, while sidestepping the design flaws of the Stoch model, we introduce a volume-based $(V-B)$ coordination mechanism. This mechanism leverages the physical coupling of electricity and natural gas systems through the GFPPs to implicitly coordinate the day-ahead and balancing markets. The system operator uses as coordination signal an amount $\chi^{v}$ of the natural gas volume that is available to GFPPs at the day-ahead stage, while the full capacity of the natural gas network is released during real-time operation. Note that volume $\chi^{v}$ affects only the fuel demand of GFPPs, while industrial/commercial natural gas loads have higher priority.

A systematic method for the definition of the optimal value of $\chi^{v}$ is mathematically formulated as the stochastic bilevel program presented schematically in Fig. 3. Similar to the Stoch model, the upper-level problem minimizes the expected cost of the integrated system, having $\chi^{v}$ as a non-negative decision variable. In turn, the lower-level problem reproduces the day-ahead clearing of the integrated market for a fixed value of $\chi^{v}$ that enters the lower level as a fixed parameter. This structure accounts for the independence of day-ahead and balancing markets, since the dayahead schedule that is enforced by the lower-level problem has the exact same properties as its counterpart in the Seq model. Consequently, the re-dispatch actions are optimized individually for each uncertainty realization in the upper level. Essentially, the optimal value of $\chi^{v}$ is found by anticipating the day-ahead market outcome and the subsequent expected balancing cost.

\footnotetext{
${ }^{3}$ Payments made to/received from market participants do not incur financial deficit to the operator.
} 


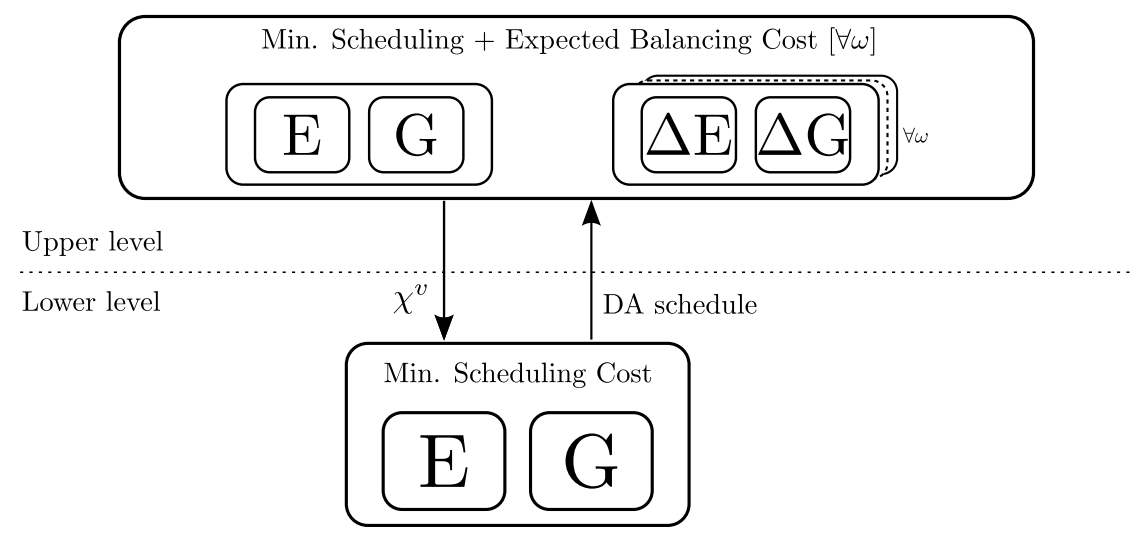

Figure 3: Volume-based coordination in sequential dispatch of integrated energy system.

Regarding the analogy with the "maximum gas burn" constraint recently adopted by CAISO, CAISO is able to limit the gas consumption of a group of generators in a defined area to increase system reliability (CAISO, 2017). In the same vein, our volume signal $\chi^{v}$ can be applied to a whole control zone or be tailored to specific areas or GFPPs. Nonetheless, the proposed mechanism extends CAISO's approach to consider primarily issues pertaining to forecast errors of stochastic power producers.

To ensure transparency for all counter-parties, the volume signal $\chi_{v}$ has to be publicly announced. When a common volume signal $\chi_{v}$ is applied to the whole control zone, no issue of discrimination is raised. However, when the volume signal $\chi_{v}$ affects specific areas or individual GFPPs, the system operator has to be aware of potential discrimination issues when only specific GFPPs are affected. It is evident that there is a trade-off between the flexibility of defining the volume signal $\chi_{v}$ and whether this is defined system-wide or area/GFPP-specific. Similar discrimination issues are raised in already established mechanisms as in the case of the "maximum gas burn constraint" imposed by CAISO during scarcity periods of natural supply. Therefore, it is always a relevant topic to be considered when dealing with reliability issues or when increasing the flexibility of the system. The definition of a systematic approach to handle this issue is out of the scope of this paper, but a potential solution could be to establish out-of-market payments to remunerate flexible producers who face remaining opportunity costs, as currently discussed in the European market context (Henriot \& Glachant, 2014).

\subsection{Price-based Coordination in Sequential Dispatch of Integrated Energy System}

Apart from the physical interaction of electricity and natural gas networks, there is also an economic link that couples the operation of these systems through the natural gas price offered to GFPPs. Therefore, a coordination mechanism analogous to the volume-based approach outlined above, can be established using instead a price-based $(P-B)$ signal $\chi^{p}$ applied to the natural gas prices. To define the optimal value of $\chi^{p}$, we employ the stochastic bilevel optimization model that is depicted schematically in Fig. 4 and proposed by Ordoudis et al. (2017). The construction of 
this model follows the same rationale as the volume-based coordination scheme and thus it also preserves the independent clearing of day-ahead and balancing markets that enforces per-se the merit-order principle.

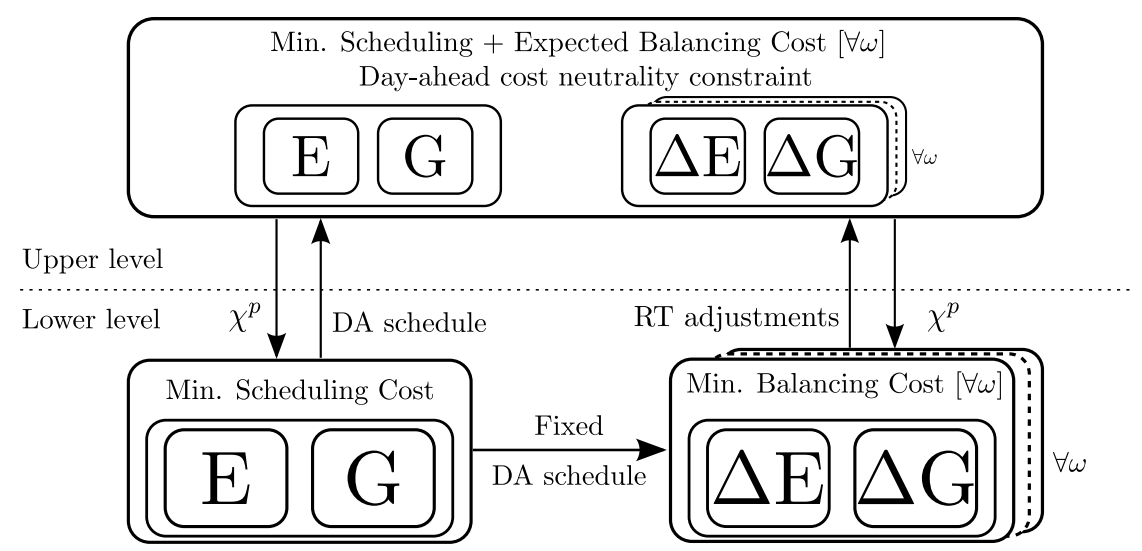

Figure 4: Price-based coordination in sequential dispatch of integrated energy system. RT: Real-time.

This coordination mechanism allows GFPPs to utilize all available natural gas resources but can instead control (either increase or decrease) the natural gas price that is perceived by the GFPPs via the free in sign $\chi^{p}$. In turn, this affects their short-term marginal costs and consequently their price offers on the electricity side of the integrated market in both day-ahead and balancing stages. Practically, these price signals reflect the scarcity value of flexible GFPPs for the system operator during real-time balancing. This scarcity value quantifies how much the system operator is willing to pay or to alter the natural gas price in order to efficiently change the merit-order of the supply curve and schedule enough flexible resources at the day-ahead stage. An efficient scheduling of the flexible resources improves the system response to the uncertainty and variability of renewable energy sources. In order to ensure non-discrimination and transparency for all counter-parties, this mechanism is designed on a cost-neutral basis such that the system operator is financially balanced at the day-ahead stage. Potential financial imbalances in the real-time settlements can be compensated using out-of-the-market payments as a supporting mechanism for the flexible producers, similar to the flexible capacity remuneration mechanisms that are currently discussed in the European electricity market context (Henriot \& Glachant, 2014).

\subsection{Features of Bilevel Models and Computational Tractability}

Models V-B and P-B are Stackelberg games (Von Stackelberg, H., 2011) where the leader (system operator) anticipates the decisions of the followers (market clearing stages). Moreover, as described in Section 2.1, the market clearing consists of two stages. The first stage is the dayahead market, while the second stage is the balancing market due to the realization of stochastic production $\omega^{\prime}$. Since the sequential arrangement between the trading floors is preserved with $V$ - $B$ and $P$ - $B$ models, cost recovery for flexible producers is guaranteed for each realization of stochastic 
production. It can be noticed that in $V-B$ implementation only the day-ahead stage needs to be included in the lower-level problem since the natural gas volume availability $\chi^{v}$ only affects the natural gas volume that is announced at the day-ahead market stage and then the physical capacity of the natural gas network is made available in real-time. In the $V$ - $B$ model, considering the outcomes of the day-ahead stage fixed to the optimal values of the sequential market design, the balancing market is settled as the last trading floor and the variables of the balancing stage do not have an impact on the solution of the day-ahead stage. Therefore, the inclusion of the balancing in the upper-level problem is equivalent to independent balancing market clearings per scenario. Alternatively, the formulation of the balancing stage could be included in the lower-level problem. This would result in the same solution but at the expense of including an additional lower-level problem per scenario $\omega$, which would increase its computational burden. On the contrary, both the day-ahead and balancing markets must be included in the lower-level problem of $P$ - $B$, since the price adjustment $\chi^{p}$ affects the marginal cost of GFPPs in the day-ahead market; however, the real-time price offers have to be altered in a consistent way to preserve the incentive for the provision of balancing services. Since the proposed dispatch models $V-B$ and $P-B$ are formulated in a bilevel structure, it is necessary to ensure that the lower-level problems are linear and convex in order to allow a single-level reformulation as a tractable mixed-integer linear program (MILP). Therefore, we study two variants of the balancing market in the following section. The first one directly permits the comparison between $V-B$ and $P-B$ since the balancing market is formulated as a linear program (LP). On the other hand, the second variant can be applied only to $V$ - $B$ as it has a detailed formulation for the gas flows in the real-time stage, which requires the introduction of binary variables that make the problem non-convex. Finally, model $V$ - $B$ results in a MILP with fewer binary variables than $P-B$ as the balancing market is not included in the lower level. Note that since the balancing market is not included in the lower level of $V$ - $B$ model, this formulation is less computationally expensive as the number of binary variables is independent of the number of scenarios. We refer the reader to (Pozo et al., 2017; Morales et al., 2014), for further discussion on bilevel optimization used in similar applications.

\section{Model Formulation}

Before presenting the mathematical formulation of the dispatch models, we introduce a set of assumptions made in this study. We consider a variation of the current market framework in which the day-ahead and balancing markets are cleared separately and at each stage the electricity and natural gas markets are co-optimized in a single optimization problem. This modeling approach allows us to assess the benefits that our models bring in terms of improved temporal coordination between the scheduling and balancing operations. Uncertain supply from stochastic producers is modeled via a finite set of scenarios $\Omega$, accounting for the temporal and spatial correlations of the forecast errors. We assume that electricity and natural gas demands are inelastic and exactly known, hence we take the operator's perspective that minimizes system's cost. The physical link 
between the electricity and natural gas systems is provided by GFPPs, where their fuel consumption has a lower priority than industrial/commercial natural gas demands. The cost structure for electricity and natural gas producers is assumed to have the form of linear functions, while stochastic producers bid at zero marginal cost. We assume a perfectly competitive market where the power producers are considered price-takers and therefore bidding at their marginal cost. We focus on the two trading floors of day-ahead and balancing markets, where a zonal-based approach, i.e. power exchange without network constraints, is used to clear the day-ahead market, while the balancing market is formulated under two different setups. The first setup is formulated as an LP under the assumption that the balancing market is cleared as a power exchange with additional fuel constraints for the GFPPs based on an ex-ante estimation of pipeline capacities, similarly to the approach used by Zhao et al. (2016). In the second setup, we introduce network constraints for both electricity and natural gas systems ${ }^{4}$. For the power system, we adopt a linearized lossless DC power flow, while a model that approximates gas flow dynamics via linepack consideration is used for the natural gas system, which leads to a MILP formulation as in (Ordoudis et al., 2019b). Note that a nomenclature is provided in the appendix.

\subsection{Sequential Dispatch of Integrated Energy System}

As illustrated in Fig. 1, the day-ahead and balancing markets are cleared independently in the Seq model. Initially, the day-ahead market is formulated in (1) as follows,

$$
\begin{aligned}
& \min _{\Theta^{\mathrm{D}}} \sum_{t \in T}\left(\sum_{i_{c} \in I_{c}} C_{i_{c}} p_{i_{c}, t}+\sum_{k \in K} C_{k} g_{k, t}\right) \\
& \text { s.t } \quad 0 \leq p_{i, t} \leq P_{i}^{\mathrm{max}}, \quad \forall i, t, \\
& 0 \leq w_{j, t} \leq \widehat{W}_{j, t}, \quad \forall j, t, \\
& 0 \leq g_{k, t} \leq G_{k}^{\mathrm{max}}, \quad \forall k, t, \\
& \sum_{i \in I} p_{i, t}+\sum_{j \in J} w_{j, t}-\sum_{r_{e} \in R_{e}} D_{r_{e}, t}^{\mathrm{E}}=0: \hat{\lambda}_{t}^{\mathrm{E}}, \quad \forall t, \\
& \sum_{k \in K} g_{k, t}-\sum_{r_{g} \in R_{g}} D_{r_{g}, t}^{\mathrm{G}}-\sum_{i_{g} \in I_{g}} \phi_{i_{g}} p_{i_{g}, t}=0: \hat{\lambda}_{t}^{\mathrm{G}}, \quad \forall t, \\
& 0 \leq \sum_{t \in T} \sum_{i_{g} \in I_{g}} \phi_{i_{g}} p_{i_{g}, t} \leq|T| \sum_{k \in K} G_{k}^{\max }-\sum_{t \in T} \sum_{r_{g} \in R_{g}} D_{r_{g}, t}^{\mathrm{G}}, \\
& 0 \leq \sum_{i_{g} \in A_{\psi}^{I_{g}}} \phi_{i_{g}} p_{i_{g}, t} \leq F_{\psi, t}^{\max }-\sum_{r_{g} \in A_{\psi}^{R_{g}}} D_{r_{g}, t}^{\mathrm{G}}, \forall \psi, t,
\end{aligned}
$$

where $\Theta^{\mathrm{D}}=\left\{p_{i, t}, \forall i, t ; w_{j, t}, \forall j, t ; g_{k, t}, \forall k, t\right\}$ is the set of optimization variables. The objective function (1a) to be minimized determines the day-ahead cost of the integrated electricity and

\footnotetext{
${ }^{4}$ The inclusion of network constraints at the balancing stage may result in higher counter-trading costs for the system operator to ensure network feasibility.
} 
natural gas system, including thermal electricity producers (i.e. excluding GFPPs) $i_{c}$ and natural gas producers $k$. Parameters $C_{i}$ and $C_{k}$ are production costs, and $t$ is the index for time periods. We have excluded the electricity cost of GFPPs $i_{g}$ since this is already accounted through the cost of their natural gas consumption. Power production $p_{i, t}$ of power plant $i$ (either thermal or GFPP) is constrained by their generation capacity $P_{i}^{\max }$ in (1b), while the power dispatch $w_{j, t}$ of the stochastic (e.g. wind) electricity producer $j$ is bounded by its expected production $\widehat{W}_{j, t}$, calculated over scenario set $\Omega$, in (1c). Moreover, natural gas production $g_{k, t}$ is constrained by the production capacity $G_{k}^{\max }$ in (1d) for each producer. The balance in power and natural gas systems is enforced through (1e) and (1f), whose dual variables $\hat{\lambda}_{t}^{\mathrm{E}}$ and $\hat{\lambda}_{t}^{\mathrm{G}}$ reflect the market price for electricity and natural gas, respectively. Note that $r_{e}$ and $r_{g}$ are indices for electricity and natural gas demands, and their loads are denoted by parameters $D_{r_{e}, t}^{\mathrm{E}}$ and $D_{r_{g}, t}^{\mathrm{G}}$. In addition, parameter $\phi_{i_{g}}$ refers to the power conversion factor for each GFPP. The marginal cost of each GFPP can be endogenously calculated by the multiplication of the natural gas price and the power conversion factor. Constraints (1g) limit the daily natural gas use of GFPPs up to the available natural gas volume at the day-ahead stage, which is determined by subtracting the commercial/industrial natural gas demand from the total daily available capacity. The number of time periods considered in one day is denoted by $|T|$. This aggregated representation allows us to conveniently control the natural gas consumption for the whole scheduling in the subsequent models using a single decision variable. We introduce a specific set to group GFPPs indexed by $\psi$, which may comprise GFPPs in a specific area of the natural gas system or even only a particular GFPP. Set $A_{\psi}^{I_{g}}$ denotes a subset of GFPPs belonging to the specific area $\psi$, while similar notation for sets $A$ is used in all formulations. The hourly fuel constraints are imposed in $(1 \mathrm{~h})$, where $F_{\psi, t}^{\max }$ denotes the maximum natural gas availability for the specific group of GFPPs. For the sake of conciseness, we denote the power adjustment provided by each power plant as $\Delta p_{i, \omega^{\prime}, t}=p_{i, \omega^{\prime}, t}^{+}-p_{i, \omega^{\prime}, t}^{-}$with $p_{i, \omega^{\prime}, t}^{+}, p_{i, \omega^{\prime}, t}^{-} \geq 0$ and the natural gas adjustment for each gas producer as $\Delta g_{k, \omega^{\prime}, t}=g_{k, \omega^{\prime}, t}^{+}-g_{k, \omega^{\prime}, t}^{-}$with $g_{k, \omega^{\prime}, t}^{+}, g_{k, \omega^{\prime}, t}^{-} \geq 0$. The day-ahead schedule is a fixed input (denoted with superscript '*') to the balancing market and model (2) simulates the balancing market to compensate for potential imbalances due to the stochastic power realization $W_{j, \omega^{\prime}, t}$, where index $\omega^{\prime}$ denotes each specific realization:

$$
\begin{aligned}
& \min _{\Theta^{\mathrm{R}}} \sum_{t \in T}\left(\sum_{k \in K}\left(C_{k}^{+} g_{k, \omega^{\prime}, t}^{+}-C_{k}^{-} g_{k, \omega^{\prime}, t}^{-}\right)+\sum_{r_{e} \in R_{e}} C^{\mathrm{sh}, \mathrm{E}} l_{r_{e}, \omega^{\prime}, t}^{\mathrm{sh}, \mathrm{E}}\right. \\
&\left.+\sum_{i_{c} \in I_{c}}\left(C_{i_{c}}^{+} p_{i_{c}, \omega^{\prime}, t}^{+}-C_{i_{c}}^{-} p_{i_{c}, \omega^{\prime}, t}^{-}\right)+\sum_{r_{g} \in R_{g}} C^{\mathrm{sh}, \mathrm{G}} l_{r_{g}, \omega^{\prime}, t}^{\mathrm{sh}, \mathrm{G}}\right) \\
& \text { s.t } \quad-p_{i, t}^{*} \leq \Delta p_{i, \omega^{\prime}, t} \leq P_{i}^{\mathrm{max}}-p_{i, t}^{*}, \quad \forall i, t, \\
& \quad-P_{i}^{-} \leq \Delta p_{i, \omega^{\prime}, t} \leq P_{i}^{+}, \quad \forall i, t, \\
& \quad 0 \leq w_{j, \omega^{\prime}, t}^{\mathrm{sp}} \leq W_{j, \omega^{\prime}, t}, \quad \forall j, t,
\end{aligned}
$$




$$
\begin{aligned}
& 0 \leq l_{r_{e}, \omega^{\prime}, t}^{\mathrm{sh}, \mathrm{E}} \leq D_{r_{e}, t}^{\mathrm{E}}, \quad \forall r_{e}, t, \\
& \sum_{i \in I} \Delta p_{i, \omega^{\prime}, t}+\sum_{r_{e} \in R_{e}} l_{r_{e}, \omega^{\prime}, t}^{\mathrm{sh}, \mathrm{E}}+\sum_{j \in J}\left(W_{j, \omega^{\prime}, t}-w_{j, \omega^{\prime}, t}^{\mathrm{sp}}-w_{j, t}^{*}\right)=0: \tilde{\lambda}_{\omega^{\prime}, t}^{\mathrm{E}}, \quad \forall t, \\
& -g_{k, t}^{*} \leq \Delta g_{k, \omega^{\prime}, t} \leq G_{k}^{\mathrm{max}}-g_{k, t}^{*}, \quad \forall k, t, \\
& -G_{k}^{-} \leq \Delta g_{k, \omega^{\prime}, t} \leq G_{k}^{+}, \quad \forall k, t, \\
& 0 \leq l_{r_{g}, \omega^{\prime}, t}^{\mathrm{sh}, \mathrm{G}} \leq D_{r_{g}, t}^{\mathrm{G}}, \quad \forall r_{g}, t, \\
& \sum_{k \in K} \Delta g_{k, \omega^{\prime}, t}+\sum_{r_{g} \in R_{g}} l_{r_{g}, \omega^{\prime}, t}^{\mathrm{sh}, \mathrm{G}}=\sum_{i_{g} \in I_{g}} \phi_{i_{g}} \Delta p_{i_{g}, \omega^{\prime}, t}: \tilde{\lambda}_{\omega^{\prime}, t}^{\mathrm{G}}, \forall t, \\
& 0 \leq \sum_{t \in T_{i_{g} \in A_{z}}^{I_{g}}} \sum_{i_{g}}\left(p_{i_{g}, t}^{*}+\Delta p_{i_{g}, \omega^{\prime}, t}\right) \leq F_{z}^{\mathrm{A}}, \quad \forall z, \\
& 0 \leq \sum_{i_{g} \in A_{z}^{I_{g}}} \phi_{i_{g}}\left(p_{i_{g}, t}^{*}+\Delta p_{i_{g}, \omega^{\prime}, t}\right) \leq F_{z, t}^{\mathrm{M}}, \quad \forall z, t, \\
& p_{i, \omega^{\prime}, t}^{+}, p_{i, \omega^{\prime}, t}^{-}, g_{k, \omega^{\prime}, t}^{+}, g_{k, \omega^{\prime}, t}^{-} \geq 0
\end{aligned}
$$

where $\Theta^{\mathrm{R}}=\left\{p_{i, \omega^{\prime}, t}^{+/-}, \forall i, t ; l_{r_{e}, \omega^{\prime}, t}^{\mathrm{sh}, \mathrm{E}}, \forall r_{e}, t ; l_{r_{g}, \omega^{\prime}, t}^{\mathrm{sh}, \mathrm{G}}, \forall r_{g}, t ; g_{k, \omega^{\prime}, t}^{+/-}, \forall k, t ; w_{j, \omega^{\prime}, t}^{\mathrm{sp}}, \forall j, t\right\}$ is the set of optimization variables. The cost of re-dispatch actions is minimized in objective function (2a). Balancing offer prices $C^{+}>C$ and $C^{-}<C$ denote the adjustment costs for thermal power plants $i_{c}$ and natural gas producers $k$, while $C^{\mathrm{sh}, \mathrm{E}}$ and $C^{\mathrm{sh}, \mathrm{G}}$ are costs for load shedding in the two systems. The bounds of power adjustments are defined in $(2 \mathrm{~b})$ considering the day-ahead dispatch of the power plants. Constraints (2c) limit power adjustments to the maximum capability $P_{i}^{+}$and $P_{i}^{-}$ of each power plant. Power spillage $w_{j, \omega^{\prime}, t}^{\mathrm{sp}}$ and electricity load shedding $l_{r_{e}, \omega^{\prime}, t}^{\mathrm{sh}, \mathrm{E}}$ are constrained by the realized power production of stochastic producers $W_{j, \omega^{\prime}, t}$ and electricity demand through (2d) and (2e), respectively. Constraints (2f) represent the power balance in real-time operation. The adjustment of natural gas production is limited by $(2 \mathrm{~g})$, where day-ahead schedules are taken into account. Additionally, constraints (2h) impose the maximum capability $G_{k}^{+}$and $G_{k}^{-}$of natural gas adjustments. Natural gas load shedding $l_{r_{e}, \omega^{\prime}, t}^{\mathrm{sh}, \mathrm{g}}$ is limited by the natural gas demand in (2i). Moreover, constraints $(2 \mathrm{j})$ impose real-time natural gas balance. The daily natural gas volume limit $F_{z}^{\mathrm{A}}$ for pipeline $z$ is imposed by $(2 \mathrm{k})$, while the real-time physical pipeline capacity $F_{z, t}^{\mathrm{M}}$ is enforced by (2l). The upper bounds of $(2 \mathrm{k})$ and (2l) are calculated based on an ex-ante analysis, where the industrial/commercial natural gas demand is subtracted by the maximum physical capacity of the pipeline and thus no explicit description of natural gas system dynamics is included.

In the remainder of the section, we present a more detailed setup for the balancing market where the network flows in electricity and natural gas systems are taken into account. At the 
electricity side, a DC power flow is considered with the following set of constraints,

$$
\begin{aligned}
& \sum_{i \in A_{n}^{I}}\left(p_{i, t}^{*}+\Delta p_{i, \omega^{\prime}, t}\right)+\sum_{r_{e} \in A_{n}^{R_{e}}} l_{r_{e}, \omega^{\prime}, t}^{\mathrm{sh} \mathrm{E}}+\sum_{j \in A_{n}^{J}}\left(W_{j, \omega^{\prime}, t}-w_{j, \omega^{\prime}, t}^{\mathrm{sp}}\right) \\
& -\sum_{r:(n, r) \in L} B_{n, r}\left(\delta_{n, \omega^{\prime}, t}-\delta_{r, \omega^{\prime}, t}\right)=\sum_{r_{e} \in A_{n}^{R_{e}}} D_{r_{e}, t}^{\mathrm{E}}: \tilde{\lambda}_{n, \omega^{\prime}, t}^{\mathrm{E}}, \forall n, t, \\
& B_{n, r}\left(\delta_{n, \omega^{\prime}, t}-\delta_{r, \omega^{\prime}, t}\right) \leq F_{n, r}^{\max }, \forall(n, r) \in L, t, \\
& \delta_{n, \omega^{\prime}, t} \text { free, } \forall n / n: \text { ref, } \delta_{n, \omega^{\prime}, t}=0, n: \text { ref, } \forall t .
\end{aligned}
$$

More specifically, the real-time balancing is imposed for each node of the power system, hence (3a) replaces (2f). Moreover, constraints (3b) determine the power flow between nodes $n$ and $r$, where $\delta_{n, \omega^{\prime}, t}$ is the voltage angle defined in (3c). The transmission capacity limits $F_{n, r}^{\max }$ are enforced by (3b).

At the gas side, an isothermal natural gas flow $q_{m, u}$ in horizontal pipelines is assumed (BorrazSanchez et al., 2016). Therefore, the Weymouth equation can be used to describe the natural gas flow from node $m$ to $u$ with the dependency at the pressure $p r_{m}$ of adjacent nodes,

$$
q_{m, u}=K_{m, u}^{f} \sqrt{p r_{m}^{2}-p r_{u}^{2}}, \forall(m, u) \in Z
$$

where $K_{m, u}^{f}$ is the Weymouth constant that depends on the physical characteristics of each pipeline $(m, u) \in Z$. Since (4) is non-linear and non-convex, we use an outer approximation by deriving the Taylor series expansion around fixed pressure points to obtain a linear expression (Tomasgard et al., 2007; Rømo et al., 2009). Consequently, we replace equality constraints (4) by the following set of linear inequalities,

$$
q_{m, u} \leq K_{m, u}^{f}\left(\frac{P R_{m, v}}{\sqrt{P R_{m, v}^{2}-P R_{u, v}^{2}}} p r_{m}-\frac{P R_{u, v}}{\sqrt{P R_{m, v}^{2}-P R_{u, v}^{2}}} p r_{u}\right), \quad \forall(m, u) \in Z, \forall v \in V .
$$

where $v \in V$ is the set of fixed pressured points $\left(P R_{m, v}, P R_{u, v}\right)$. To ensure an efficient approximation of the equation (4), we use a large number of fixed pressure points $\left(P R_{m, v}, P R_{u, v}\right)$, as proposed by Fodstad et al. (2015). Thus, we achieve an outer approximation by the constructed planes in (5) that are tangent to the surface defined by the Weymouth equation in (4). This results in approximating the gas flow by the single linear constraint in (5) that is binding (Tomasgard et al., 2007). An advanced natural gas system with linepack is modeled using the following constraints,

$$
\begin{aligned}
& P R_{m}^{\min } \leq p r_{m, \omega^{\prime}, t} \leq P R_{m}^{\max }, \quad \forall m, t \\
& p r_{u, \omega^{\prime}, t} \leq \Gamma_{z} \cdot p r_{m, \omega^{\prime}, t}, \quad, \forall(m, u) \in Z, t, \\
& q_{m, u, \omega^{\prime}, t}=q_{m, u, \omega^{\prime}, t}^{+}-q_{m, u, \omega^{\prime}, t}^{-}, \quad \forall(m, u) \in Z, t,
\end{aligned}
$$




$$
\begin{aligned}
& 0 \leq q_{m, u, \omega^{\prime}, t}^{+} \leq \tilde{M}_{m, u} y_{m, u, \omega^{\prime}, t}, \quad \forall(m, u) \in Z, t, \\
& 0 \leq q_{m, u, \omega^{\prime}, t}^{-} \leq \tilde{M}_{m, u}\left(1-y_{m, u, \omega^{\prime}, t}\right), \quad \forall(m, u) \in Z, t, \\
& y_{m, u, \omega^{\prime}, t}+y_{u, m, \omega^{\prime}, t}=1, \quad \forall(m, u) \in Z, t, \\
& y_{m, u, \omega^{\prime}, t} \in\{0,1\}, \quad \forall(m, u) \in Z, t, \\
& q_{m, u, \omega^{\prime}, t}^{+} \leq K I_{m, u, v}^{+} p r_{m, \omega^{\prime}, t}-K O_{m, u, v}^{+} p r_{u, \omega^{\prime}, t}+\tilde{M}_{m, u}\left(1-y_{m, u, \omega^{\prime}, t}\right), \forall\{(m, u) \in Z \mid m<u\}, \forall v, t \\
& q_{m, u, \omega^{\prime}, t}^{-} \leq K I_{m, u, v}^{-} p r_{u, \omega^{\prime}, t}-K O_{m, u, v}^{-} p r_{m, \omega^{\prime}, t}+\tilde{M}_{m, u} y_{m, u, \omega^{\prime}, t}, \forall\{(m, u) \in Z \mid m<u\}, \forall v, t, \\
& q_{u, m, \omega^{\prime}, t}^{-} \leq K I_{m, u, v}^{+} p r_{m, \omega^{\prime}, t}-K O_{m, u, v}^{+} p r_{u, \omega^{\prime}, t}+\tilde{M}_{m, u} y_{u, m, \omega^{\prime}, t}, \forall\{(m, u) \in Z \mid m<u\}, \forall v, t, \\
& q_{u, m, \omega^{\prime}, t}^{+} \leq K I_{m, u, v}^{-} p r_{u, \omega^{\prime}, t}-K O_{m, u, v}^{-} p r_{m, \omega^{\prime}, t}+\tilde{M}_{m, u}\left(1-y_{u, m, \omega^{\prime}, t}\right), \forall\{(m, u) \in Z \mid m<u\}, \forall v, t, \\
& q_{m, u, \omega^{\prime}, t}^{+}=\frac{q_{m, u, \omega^{\prime}, t}^{\mathrm{in}}+q_{m, u, \omega^{\prime}, t}^{\mathrm{out}}}{2}, \forall(m, u) \in Z, t, \\
& q_{m, u, \omega^{\prime}, t}^{-}=\frac{q_{u, m, \omega^{\prime}, t}^{\mathrm{in}}+q_{u, m, \omega^{\prime}, t}^{\mathrm{out}}}{2}, \forall(m, u) \in Z, t, \\
& h_{m, u, \omega^{\prime}, t}=K_{m, u}^{\mathrm{h}} \frac{p r_{m, \omega^{\prime}, t}+p r_{u, \omega^{\prime}, t}}{2}, \forall(m, u) \in Z, t, \\
& h_{m, u, \omega^{\prime}, t}=h_{m, u, \omega^{\prime}, t-1}+q_{m, u, \omega^{\prime}, t}^{\text {in }}-q_{m, u, \omega^{\prime}, t}^{\text {out }}, \forall(m, u) \in Z, t, \\
& \sum_{k \in A_{m}^{K}}\left(g_{k, t}^{*}+\Delta g_{k, \omega^{\prime}, t}\right)+\sum_{r_{g} \in A_{m}^{R g}} l_{r_{g}, \omega^{\prime}, t}^{\mathrm{sh}, \mathrm{G}}-\sum_{i_{g} \in A_{m}^{I_{g}}} \phi_{i_{g}}\left(p_{i, t}^{*}+\Delta p_{i_{g}, \omega^{\prime}, t}\right) \\
& -\sum_{u:(m, u) \in Z}\left(q_{m, u, \omega^{\prime}, t}^{\mathrm{in}}-q_{u, m, \omega^{\prime}, t}^{\mathrm{out}}\right)=\sum_{r_{g} \in A_{m}^{R_{g}}} D_{r_{g}, t}^{\mathrm{G}}: \tilde{\lambda}_{m, \omega^{\prime}, t}^{\mathrm{G}}, \forall m, t . \\
& q_{u, m, \omega^{\prime}, t}^{\text {in }}, q_{u, m, \omega^{\prime}, t}^{\text {out }} \geq 0
\end{aligned}
$$

The bounds of pressure at each node of the system $P R_{m}^{\min }$ and $P R_{m}^{\max }$ are given by (6a), while the active pipelines are modeled by the relation of pressures between the two adjacent nodes via a compression factor $\Gamma_{z}$ in (6b), in order to model compressors in the natural gas system (CorreaPosada \& Sanchez-Martin, 2014). More specifically, the outlet pressure at node $u$ is greater than the inlet pressure at node $m$, when the gas flow is from $m$ to $u$ for the active branches. The natural gas flow $q_{m, u, \omega^{\prime}, t}$ is defined in (6c)-(6g) by two non-negative variables $q_{m, u, \omega^{\prime}, t}^{+}, q_{m, u, \omega^{\prime}, t}^{-} \geq 0$, where the direction of flow is defined by the binary variable $y_{u, m, \omega^{\prime}, t}$. Note that parameter $\tilde{M}_{m, u}$ is a sufficient large constant that reflects the maximum physical capacity of each pipeline. The physical characteristics of gas flow are introduced in (6h)-(6k) that are derived by (5) with

$$
\begin{aligned}
& \left\{K I_{m, u, v}^{+}=\frac{K_{m, u}^{f} P R_{m, v}}{\sqrt{P R_{m, v}^{2}-P R_{u, v}^{2}}}, K O_{m, u, v}^{+}=\frac{K_{m, u}^{f} P R_{u, v}}{\sqrt{P R_{m, v}^{2}-P R_{u, v}^{2}}},\right. \\
& \left.K I_{m, u, v}^{-}=\frac{K_{m, u}^{f} P R_{u, v}}{\sqrt{P R_{u, v}^{2}-P R_{m, v}^{2}}}, K O_{m, u, v}^{-}=\frac{K_{m, u}^{f} P R_{m, v}}{\sqrt{P R_{u, v}^{2}-P R_{m, v}^{2}}}\right\}, \forall\{(m, u) \in Z \mid m<u\}, \forall v .
\end{aligned}
$$


Finally, two additional non-negative variables for the inflow and outflow of each pipeline $q_{u, m, \omega^{\prime}, t}^{\text {in }}$, $q_{u, m, \omega^{\prime}, t}^{\text {out }} \geq 0$ are introduced to model linepack flexibility. Constraints (61) and (6m) define the flow of each pipeline as the average of inflow and outflow (Correa-Posada \& Sanchez-Martin, 2014). The average mass $h_{m, u, \omega^{\prime}, t}$ in each pipeline is given by $(6 \mathrm{n})$, where $K_{m, u}^{\mathrm{h}}$ is a constant dependent on pipeline characteristics. The mass conservation at each pipeline is enforced by (6o). The natural gas balancing in the real-time is enforced by $(6 \mathrm{p})$ that replaces $(2 \mathrm{j})$. The presented model approximates the dynamics of the natural gas system and is described in detail in (Ordoudis et al., 2019b; Schwele et al., 2019), while a schematic representation to facilitate the relationship among the variables of the natural gas flow is provided in the online appendix (Ordoudis et al., 2019a). We refer the reader to (Misra et al., 2015) for a steady-state modeling of the natural gas system with geometric programming and to (Zlotnik et al., 2015) for a transient model that closely describes the physical behaviour of the natural gas flow. Alternative technologies, such as electricity storage, underground natural gas storage and flexible demand, can be incorporated into the model in a straightforward manner. In this work, the focus is placed only to natural gas storage via linepack and therefore such alternative technologies are left for future extensions.

The zonal-based balancing market is formulated with the set of constraints (2b)-(2l). A different set of constraints is used for the network constrained balancing market that consists of $\{(2 \mathrm{~b})-(2 \mathrm{e}),(2 \mathrm{~g})-(2 \mathrm{i}),(3 \mathrm{a})-(3 \mathrm{c}),(6 \mathrm{a})-(6 \mathrm{p})\}$. In both cases, the objective function is (2a). We use " $N$ " to determine the use of network-constrained balancing market, hence models $S e q$ and $S e q-N$ are formulated. Moreover, the set of primary variables $\Theta^{\mathrm{R}}$ is extended with $\Theta^{\mathrm{EX}}=\left\{\delta_{n, \omega^{\prime}, t}, \forall n, t\right.$; $\left.p r_{m, \omega^{\prime}, t} \forall m, t ; q_{m, u, \omega^{\prime}, t}^{\text {in }}, q_{m, u, \omega^{\prime}, t}^{+/-}, h_{m, u, \omega^{\prime}, t}, y_{u, m, \omega^{\prime}, t}, \forall(m, u) \in Z, t\right\}$ in $S e q-N$. In this model, the electricity and natural gas prices are derived as the dual variables of the balance constraints of the LP obtained by fixing the binary variables related to the natural gas flow direction of the MILP to the optimal values. A potential caveat of this approach, due to the non-convexity of the original MILP, is that the resulting prices may not adequately support the economic dispatch. In that case, corrective out-of-market payments may be considered, e.g. similar to uplift payments ${ }^{5}$ in the unit commitment problem, to provide the right incentives to follow the dispatch instructions (Gribik et al., 2007; O'Neill et al., 2005). The expected balancing cost over a scenario set $\Omega$ is given as the sum of the balancing cost for each scenario $\omega$ weighed by its probability of occurrence $\pi_{\omega}$.

\subsection{Stochastic Dispatch of Integrated Energy System}

As presented in Fig. 2, the Stoch model optimizes jointly the day-ahead and balancing stages of the integrated electric power and natural gas systems. The problem is formulated as a two-stage

\footnotetext{
${ }^{5}$ Payments from the operator to ensure that the profit of each producer is non-negative. This mechanism is typically used in case when generation schedules are derived from a unit commitment. In that case, energy prices based on marginal costs are not able to support producers in recovering their total cost, since costs modelled by non-convex binary constraints, such that start-up/shut-down costs, are not reflected in the energy prices.
} 
stochastic program aiming to minimize the total expected cost and writes as follows,

$$
\begin{aligned}
\min _{\Theta^{\mathrm{SC}}} & \sum_{t \in T}\left[\sum_{i_{c} \in I_{c}} C_{i_{c}} p_{i_{c}, t}+\sum_{k \in K} C_{k} g_{k, t}+\sum_{\omega \in \Omega} \pi_{\omega}\left(\sum_{k \in K}\left(C_{k}^{+} g_{k, \omega, t}^{+}-C_{k}^{-} g_{k, \omega, t}^{-}\right)\right.\right. \\
& \left.\left.+\sum_{i_{c} \in I_{c}}\left(C_{i_{c}}^{+} p_{i_{c}, \omega, t}^{+}-C_{i_{c}}^{-} p_{i_{c}, \omega, t}^{-}\right)+\sum_{r_{e} \in R_{e}} C^{\mathrm{sh}, \mathrm{E}} l_{r_{e}, \omega, t}^{\mathrm{sh}, \mathrm{E}}+\sum_{r_{g} \in R_{g}} C^{\mathrm{sh}, \mathrm{G}} l_{r_{g}, \omega, t}^{\mathrm{sh}, \mathrm{G}}\right)\right] \\
\text { s.t } & \text { constraints }(1 \mathrm{~b}),(1 \mathrm{~d})-(1 \mathrm{~h}), \\
& 0 \leq w_{j, t} \leq \bar{W}_{j}, \forall j, t, \\
& \text { constraints }(2 \mathrm{~b})-(2 \mathrm{l}), \quad \forall \omega,
\end{aligned}
$$

where $\Theta^{\mathrm{SC}}=\left\{\Theta^{\mathrm{D}} ; \Theta_{\omega}^{\mathrm{R}}, \forall \omega\right\}$ is the set of optimization variables. In this model, the temporal coordination of the two trading floors is achieved through the real-time constraints (8d) for all scenarios $\omega \in \Omega$. When network constrains $\{(2 \mathrm{~b})-(2 \mathrm{e}),(2 \mathrm{~g})-(2 \mathrm{i}),(3 \mathrm{a})-(3 \mathrm{c}),(6 \mathrm{a})-(6 \mathrm{p})\}$ are introduced to replace (8d), the model is named Stoch- $N$. Note that in model (8), the day-ahead dispatch of stochastic producers is restricted by the installed capacity $\bar{W}_{j}$, according to (8c), instead of the expected power generation and day-ahead dispatch decisions are treated as variables.

\subsection{Volume-based Coordination in Sequential Dispatch of Integrated Energy System}

According to Fig. 3, the $V$ - $B$ dispatch model that aims at minimizing the expected cost of the integrated energy system and defining the optimal natural gas volume availability writes as follows,

$$
\begin{gathered}
\min _{\Theta \mathrm{VUL}}(8 \mathrm{a}) \\
\text { s.t }(2 \mathrm{~b})-(2 \mathrm{l}), \quad \forall \omega, \\
0 \leq \bar{\chi}_{\psi}^{v} \leq|T| \sum_{k \in K} G_{k}^{\mathrm{max}}-\sum_{t \in T} \sum_{r_{g} \in R_{g}} D_{r_{g}, t}^{\mathrm{G}}, \quad \forall \psi, \\
0 \leq \chi_{\psi, t}^{v} \leq F_{\psi, t}^{\mathrm{max}}-\sum_{r_{g} \in A_{\psi}^{R_{g}}} D_{r_{g}, t}^{\mathrm{G}}, \quad \forall \psi, t, \\
\left(p_{i, t}, w_{j, t}, g_{k, t}\right) \in \arg \left\{\sum_{\Theta^{\mathrm{VLL}}} \sum_{t \in T}\left(\sum_{i_{c} \in I_{c}} C_{i_{c}} p_{i_{c}, t}+\sum_{k \in K} C_{k} g_{k, t}\right)\right. \\
\operatorname{s.t} \quad \operatorname{constraints}(1 \mathrm{~b})-(1 \mathrm{f}), \\
0 \leq \sum_{t \in T} \sum_{i_{g} \in A_{\psi}^{I_{g}}} \phi_{i_{g}} p_{i_{g}, t} \leq \bar{\chi}_{\psi}^{v}, \quad \forall \psi, \\
\left.0 \leq \sum_{i_{g} \in A_{\psi}^{I_{g}}} \phi_{i_{g}} p_{i_{g}, t} \leq \chi_{\psi, t}^{v}, \quad \forall \psi, t\right\},
\end{gathered}
$$


where $\Theta^{\mathrm{VUL}}=\left\{\bar{\chi}_{\psi}^{v}, \forall \psi ; \chi_{\psi, t}^{v}, \forall \psi, t ; \Theta_{\omega}^{\mathrm{R}}, \forall \omega\right\}$ is the set of optimization variables of the upper-level problem. Additionally, $\Theta^{\mathrm{VLL}}=\Theta^{\mathrm{D}}$ is the set of optimization variables of the lower-level problem. The objective function of model (9) is the same as in (8). Thus, the upper-level problem minimizes the expected cost of operating the integrated energy system by deciding the optimal value of $\bar{\chi}_{\psi}^{v}$ and $\chi_{\psi, t}^{v}$. Variable $\bar{\chi}_{\psi}^{v}$ limits the total daily natural gas consumption of GFPPs according to (9g), while $\chi_{\psi, t}^{v}$ defines their hourly fuel limit in $(9 \mathrm{~h})$. We define fuel availability $\bar{\chi}_{\psi}^{v}$ and $\chi_{\psi, t}^{v}$ under different setups, ranging from a single value for the whole market to individual values for specific areas or GFPPs. Therefore, these two variables are indexed by $\psi \in \Psi$ that defines the GFPPs that are grouped together in each setup. The lower-level problem reproduces the day-ahead coupled electricity and natural gas market. Under this setup the sequential clearing of day-ahead and balancing markets is practically emulated, since the day-ahead decisions are fixed to the sequential dispatch though (9e)-(9h) and the balancing market is simulated for each independent scenario by imposing constraints (9b) for all $\omega \in \Omega$. The system operator has the ability to decide the natural gas volume that will be made available for power production at the day-ahead stage within specified limits, defined by (9c) and (9d).Note that (9c) and (9d) resemble constraints (1g) and (1h) of Seq dispatch model. The upper-level variables $\bar{\chi}_{\psi}^{v}$ and $\chi_{\psi, t}^{v}$ have an impact on the decisions of the lower-level problem as the total fuel availability for GFPPs affects the day-ahead schedule for power production. Moreover, the lower-level decision variables affect the total expected cost of the integrated system. Owning to this structure, model (9) finds an appropriate dispatch that minimizes expected system cost by revealing flexibility from GFPPs, while ensuring that the leastcost merit-order principle is respected. The different effects of these approaches are illustrated in the numerical study. Similarly, the model is named $V-B-N$ with the introduction of network constrains $\{(2 \mathrm{~b})-(2 \mathrm{e}),(2 \mathrm{~g})-(2 \mathrm{i}),(3 \mathrm{a})-(3 \mathrm{c}),(6 \mathrm{a})-(6 \mathrm{p})\}$ to replace $(9 \mathrm{~b})$. The bilevel problem (9) can be reformulated as a Mathematical Program with Equilibrium Constraints (MPEC) by replacing the linear, and thus convex, lower-level problems by their Karush-Kuhn-Tucker (KKT) conditions as presented in the online appendix (Ordoudis et al., 2019a).

\subsection{Price-based Coordination in Sequential Dispatch of Integrated Energy System}

As shown in Fig. 4, the $P-B$ dispatch model minimizes the expected cost of the integrated energy system and defines the optimal natural gas price adjustment. Model $P-B$ writes as follows,

$$
\begin{array}{ll}
\min _{\Theta^{\mathrm{PUL}}} & (8 \mathrm{a}) \\
\text { s.t } & -X \leq \chi_{t}^{p} \leq X, \quad \forall t, \\
& \sum_{t \in T} \sum_{i_{g} \in I_{g}} \phi_{i_{g}} p_{i_{g}, t} \chi_{t}^{p}=0,
\end{array}
$$




$$
\begin{aligned}
& \left(p_{i, t}, w_{j, t}\right) \in \arg \{ \\
& \min _{\Theta_{1}^{\mathrm{PLL}}} \sum_{t \in T}\left(\sum_{i_{c} \in I_{c}} C_{i_{c}} p_{i_{c}, t}+\sum_{i_{g} \in I_{g}} C_{i_{g}, t} p_{i_{g}, t}\right) \\
& \text { s.t constraints }(1 \mathrm{~b}),(1 \mathrm{c}),(1 \mathrm{e}),(1 \mathrm{~g}),(1 \mathrm{~h}) \text {, } \\
& \left.C_{i_{g}, t}=\left(\hat{\lambda}_{t}^{\mathrm{G}}+x_{t}\right) \phi_{i_{g}}, \forall i_{g}, t\right\} \text {, } \\
& \left(p_{i, \omega, t}^{+}, p_{i, \omega, t}^{-}, w_{j, \omega, t}^{\mathrm{sp}}, l_{\omega, t}^{\mathrm{sh}, \mathrm{E}}\right) \in \arg \{ \\
& \min _{\Theta_{2}^{\mathrm{PLL}}} \sum_{t \in T}\left(\sum_{i_{c} \in I_{c}}\left(C_{i_{c}}^{+} p_{i_{c}, \omega, t}^{+}-C_{i_{c}}^{-} p_{i_{c}, \omega, t}^{-}\right)+\sum_{i_{g} \in I_{g}}\left(C_{i_{g}, \omega, t}^{+} p_{i_{g}, \omega, t}^{+}-C_{i_{g}, \omega, t}^{-} p_{i_{g}, \omega, t}^{-}\right)\right. \\
& \left.+C^{\mathrm{sh}, \mathrm{E}} l_{\omega, t}^{\mathrm{sh}, \mathrm{E}}+\sum_{j \in J} C^{\mathrm{sp}} w_{j, \omega, t}^{\mathrm{sp}}\right) \\
& \text { s.t constraints }(2 \mathrm{~b})-(2 \mathrm{f}),(2 \mathrm{k}),(2 \mathrm{l}) \text {, } \\
& C_{i_{g}, \omega, t}^{+}=\left(\tilde{\lambda}_{\omega, t}^{\mathrm{G}}+x_{t}\right) \phi_{i_{g}}, \quad \forall i_{g}, t \\
& \left.C_{i_{g}, \omega, t}^{-}=\left(\tilde{\lambda}_{\omega, t}^{\mathrm{G}}+x_{t}\right) \phi_{i_{g}}, \forall i_{g}, t\right\}, \forall \omega, \\
& \left(g_{k, t}, \hat{\lambda}_{t}^{\mathrm{G}}\right) \in \arg \{ \\
& \min _{\Theta_{3}^{\mathrm{PLL}}} \sum_{t \in T} \sum_{k \in K} C_{k} g_{k, t} \\
& \text { s.t constraints (1d), (1f) }\} \text {, } \\
& \left(g_{k, \omega, t}^{+}, g_{k, \omega, t}^{-}, l_{\omega, t}^{\mathrm{sh}, \mathrm{G}}, \tilde{\lambda}_{\omega, t}^{G}\right) \in \arg \{ \\
& \left.\min _{\Theta_{4}^{\mathrm{PLL}}} \sum_{t \in T}\left(\sum_{k \in K}\left(C_{k}^{+} g_{k, \omega, t}^{+}-C_{k}^{-} g_{k, \omega, t}^{-}\right)\right)+C^{\mathrm{sh}, \mathrm{G}} l_{\omega, t}^{\mathrm{sh}, \mathrm{G}}\right) \\
& \text { s.t constraints }(2 \mathrm{~g})-(2 \mathrm{j})\}, \forall \omega \text {, }
\end{aligned}
$$

where $\Theta_{1}^{\mathrm{PLL}}=\left\{p_{i, t}, \forall i, t ; w_{j, t}, \forall j, t\right\}, \Theta_{2}^{\mathrm{PLL}}=\left\{p_{i, \omega, t}^{+}, p_{i, \omega, t}^{-}, \forall i, \omega, t ; l_{\omega, t}^{\mathrm{sh}, \mathrm{E}}, \forall \omega, t ; w_{j, \omega, t}^{\mathrm{sp}}, \forall j, \omega, t\right\}$, $\Theta_{3}^{\mathrm{PLL}}=\left\{g_{k, t}, \forall k, t\right\}$ and $\Theta_{4}^{\mathrm{PLL}}=\left\{g_{k, \omega, t}^{+}, g_{k, \omega, t}^{-}, \forall k, \omega, t ; l_{\omega, t}^{\text {sh, }}, \forall \omega, t\right\}$ are the sets of optimization variables of the lower-level problems. Additionally, $\Theta^{\mathrm{PUL}}=\left\{\chi_{t}^{p}, \forall t\right\}$ is the set of optimization variables of the upper-level problem. The upper-level problem minimizes the total expected cost and optimally decides the value of natural gas price adjustment $\chi_{t}^{p}$. The lower-level problems practically simulate the sequential dispatch of day-ahead and balancing stages, since the day-ahead decisions are fixed to the sequential dispatch via (10d)-(10f) and (10k)-(10l), while the balancing stage is simulated for each scenario $\omega \in \Omega$ by $(10 \mathrm{~g})-(10 \mathrm{j})$ and $(10 \mathrm{~m})-(10 \mathrm{n})$. The natural gas price adjustment is bounded in constraints (10b) which indicates the limits that the operator is able to vary the natural gas price perceived by GFPPs. These bounds can be set either wide enough so that the natural gas price adjustments could be able to move the GFPPs to any position of the supply curve or in a way that the operator will only have a smaller interference to the natural 
gas price and hence to the marginal cost of GFPPs. Equality (10c) enforces cost-neutrality at the day-ahead stage guaranteeing that the operator will not have a financial deficit or surplus throughout the scheduling horizon. Note that any potential deficit or surplus at the balancing stage is expected to be reasonably small and can be addressed via proper regulation. The upper-level variable $\chi_{t}^{p}$ affects the decisions of lower-level problems by the day-ahead and balancing offer prices of GFPPs via equality constraints (10f), (10i) and (10j), which in turn influences the objective functions $(10 \mathrm{~d}),(10 \mathrm{~g}),(10 \mathrm{k})$ and $(10 \mathrm{~m})$ in the lower-level problems. Moreover, the dispatch of GFPPs affects the values of lower-level decision variables which then have an impact on the total expected cost of the coupled energy system. Hence, model (10) provides the optimal system schedule that anticipates future balancing costs and simultaneously respects the least-cost merit-order principle. As discussed in Section 2.5, model $P$ - $B$ is not formulated with network constraints $\{(2 \mathrm{~b})-(2 \mathrm{e}),(2 \mathrm{~g})-$ (2i),(3a)-(3c),(6a)-(6p)\}, since the lower-level problems would include binary variables that make them non-convex. Similarly to bilevel problem (9), bilevel problem (10) can be reformulated as an MPEC as presented by Ordoudis et al. (2017).

\subsection{Overview of Dispatch Models}

Before proceeding to the numerical results, we provide an overview of the dispatch models and their features in Table 1.

Table 1: Dispatch models' characteristics

\begin{tabular}{ccccc}
\hline $\begin{array}{c}\text { Fuel / Network } \\
\text { constraints }\end{array}$ & Seq / Seq-N & Stoch / Stoch- $N$ & $V-B / V-B-N$ & $P-B /-$ \\
\hline $\begin{array}{c}\text { Temporal } \\
\text { coordination } \\
\begin{array}{c}\text { Coordination } \\
\text { mechanism }\end{array}\end{array}$ & Imperfect & Non-existing & Explicit & Implicit via $\chi^{v}{ }^{*}$ Implicit via $\chi^{p}$ \\
\hline${ }^{*}$ The value of $\chi^{v}$ can be defined for the whole market, specific areas or GFPPs.
\end{tabular}

The dispatch models are classified based on the networks' representation in the balancing market, as well as the temporal coordination achieved and the mechanism utilized.

\section{Numerical Results}

In this section, we first demonstrate the features of the four dispatch models presented in Section 2 in a tailored case study. Then, we compare the performance of the volume-based variants on a more realistic case study.

\subsection{Tailored Case-Study}

\subsubsection{1-hour Simulation Results}

To allow a fair comparison between $P-B$ and $V$ - $B$ models, the balancing market in this illustrative example is modeled as a power exchange with fuel constraints for GFPPs. Moreover, we 
assume a single type of uncertain supply that is wind power. Here, we consider a system which comprises three thermal power plants $\left(I_{1}, I_{2}\right.$ and $\left.I_{3}\right)$, two GFPPs $\left(I_{4}\right.$ and $\left.I_{5}\right)$ that acquire their fuel from the natural gas market, one wind farm (WP) and two natural gas producers $\left(K_{1}\right.$ and $K_{2}$ ). Table 2 collects the data for the producers in both markets. The wind farm has a capacity of $215 \mathrm{MW}$ and the wind power production is characterized by two equiprobable scenarios $\omega_{1}$ (166 $\mathrm{MW})$ and $\omega_{2}(86 \mathrm{MW})$. The offer prices for upward and downward regulation are equal to 1.1 and 0.9 of the day-ahead offer prices. In $P$ - $B$, we limit the natural gas price adjustment to $\$ 1.35 / \mathrm{kcf}$. Moreover, we consider a pipeline capacity of $6,000 \mathrm{kcf}$. The cost of electricity and natural gas load shedding is $\$ 1,200 / \mathrm{MWh}$ and $\$ 600 / \mathrm{kcf}$, while wind spillage is cost-free. The peak electricity and natural gas demand for industrial/commercial loads are equal to $430 \mathrm{MW}$ and 3,600 kcf/h, respectively.

Table 2: Electric power and natural gas system data (kcf: kilo cubic feet)

\begin{tabular}{lccccc|lcc}
\hline Unit $i$ & $I_{1}$ & $I_{2}$ & $I_{3}$ & $I_{4}$ & $I_{5}$ & Unit $k$ & $K_{1}$ & $K_{2}$ \\
\hline$P_{i}^{\max }(\mathrm{MW})$ & 110 & 80 & 100 & 50 & 100 & $G_{k}^{\max }(\mathrm{kcf})$ & 10,000 & 6,000 \\
$P_{i}^{+}(\mathrm{MW})$ & 0 & 10 & 20 & 30 & 25 & $G_{k}^{+}(\mathrm{kcf})$ & 2,500 & 1,000 \\
$P_{i}^{-}(\mathrm{MW})$ & 0 & 10 & 20 & 30 & 25 & $G_{k}^{-}(\mathrm{kcf})$ & 2,500 & 1,000 \\
$C_{i}(\$ / \mathrm{MWh})$ & 10 & 30 & 60 & - & - & $C_{k}(\$ / \mathrm{kcf})$ & 2 & 3 \\
$\phi_{i_{g}}(\mathrm{kcf} / \mathrm{MWh})$ & - & - & - & 12 & 18 & & & \\
\hline
\end{tabular}

We solve all dispatch models for 24-hours and provide detailed results for a specific time period, where $\chi^{p}$ has a negative value. Additional results for the case that $\chi^{\mathrm{p}}$ has a positive value are included in the online appendix (Ordoudis et al., 2019a). Two variants of (9), where natural gas volume availability is determined for the whole market $(V-B)$ and for each individual GFPP ( $V$ - $B$ gen), are studied. In this specific time period and for all dispatch models, natural gas is produced only by unit $K_{1}$, hence the natural gas price is $\$ 2 / \mathrm{kcf}$ and the marginal costs of GFPPs $I_{4}$ and $I_{5}$ are $\$ 24 / \mathrm{MWh}$ and $\$ 36 / \mathrm{MWh}$.

The action of adjusting the natural gas price or volume availability becomes beneficial when the day-ahead dispatch is altered with regards to $S e q$ to allow more cost-effective power plants to provide the required regulation in the balancing market. This results in an increase of the day-ahead cost and a decrease of expected balancing cost that yields a reduction of total expected cost.

Initially, we demonstrate the performance of all dispatch models in Table 3, when the total electricity demand is equal to $387 \mathrm{MW}$ and the wind power penetration level is $55.5 \%$, defined as the share of wind power capacity on total system's electricity demand. It can be observed that Stoch returns the lowest expected system cost and $S e q$ the highest one due to imperfect temporal coordination between day-ahead and balancing markets. Models $P-B, V-B$ and $V$ - $B$ gen attain an expected cost that is bounded by the ideal solution of Stoch and the one of Seq. Thus, a reduction of expected system cost can be accomplished, while the system is still dispatched based on the least-cost merit-order principle. 
Table 3: Expected system cost and its breakdown in $\$$ when total power load is $387 \mathrm{MW}$

\begin{tabular}{cccccc}
\hline & Total & Day-ahead & Balancing & Up regulation & Down regulation \\
\hline Seq / $V$ - $B$ & 10,400 & 9,982 & 418 & 990 & -572 \\
Stoch & 10,234 & 10,222 & 12 & 660 & -648 \\
$P-B$ & 10,273 & 10,042 & 231 & 825 & -594 \\
$V$ - B gen & 10,261 & 10,162 & 99 & 693 & -594 \\
\hline
\end{tabular}

The detailed system dispatch is illustrated in Table 4 for an electricity demand of 387 MW. Regarding $P-B$, the marginal cost of all GFPPs is affected by adjusting the natural gas price with $\chi_{t_{1}}^{p}=-\$ 0.333 / \mathrm{kcf}$. The decreased natural gas price of $\$ 1.666 / \mathrm{kcf}$ results in a lower marginal cost for GFPP $I_{5}$ equal to $\$ 30 / \mathrm{MWh}$, which equals the one of unit $I_{2}$. Therefore, unit $I_{2}$ is dispatched to $70 \mathrm{MW}$ and GFPP $I_{5}$ to $31 \mathrm{MW}$ without breaking the merit order. Model $V$ - $B$ returns the same results with $S e q$, as a change of total natural gas volume availability would not decrease the total expected cost. On the contrary, $V-B$ gen has a better performance due to its ability to influence the dispatch of both GFPPs $I_{4}$ and $I_{5}$. Note that GFPPs $I_{4}$ and $I_{5}$ produce a total of $71 \mathrm{MW}$ in both $S e q$ and $V$-B gen at the day-ahead stage. However, the allocation between the two GFPPs is different and more efficient under $V$-B gen. More specifically, the total natural gas volume bought by GFPPs in $S e q$ is $987 \mathrm{kcf}$, where $600 \mathrm{kcf}$ are consumed by GFPP $I_{4}$ and the remaining $387 \mathrm{kcf}$ by GFPP $I_{5}$. In $V$-B gen, the natural gas volume made available for GFPP $I_{4}$ is $420 \mathrm{kcf}$, while GFPP $I_{5}$ consumes $648 \mathrm{kcf}$. The adjustment of natural gas volume availability has a direct impact on the day-ahead dispatch which in turn reduces the total expected cost compared to Seq. The day-ahead cost increases but this increase is counterbalanced by a greater decrease of balancing cost. In particular, the up-regulation cost is decreased because unit $I_{3}$ is not activated and the need for up-regulation is covered by the cheaper GFPP $I_{4}$. Moreover, a greater portion of the total $40 \mathrm{MW}$ needed for down-regulation is provided by GFPP $I_{5}$ that is more cost-effective than GFPP $I_{4}$.

Table 4: Power system schedule in MW when total power load is $387 \mathrm{MW}$ (variation from Seq day-ahead (DA) schedule in bold)

\begin{tabular}{c|ccc|ccc|ccc|ccc|ccc}
\hline & \multicolumn{3}{|c|}{$S e q$} & \multicolumn{3}{|c|}{$P-B$} & \multicolumn{3}{c|}{$V-B$} & \multicolumn{3}{c}{$V$ - $B$ gen } & \multicolumn{3}{c}{ Stoch } \\
\hline Unit & DA & $\omega_{1}$ & $\omega_{2}$ & DA & $\omega_{1}$ & $\omega_{2}$ & DA & $\omega_{1}$ & $\omega_{2}$ & DA & $\omega_{1}$ & $\omega_{2}$ & DA & $\omega_{1}$ & $\omega_{2}$ \\
\hline$I_{1}$ & 110 & 0 & 0 & 110 & 0 & 0 & 110 & 0 & 0 & 110 & 0 & 0 & 110 & 0 & 0 \\
$I_{2}$ & 80 & -10 & 0 & $\mathbf{7 0}$ & -10 & +10 & 80 & -10 & 0 & 80 & -10 & 0 & 70 & -10 & +10 \\
$I_{3}$ & 0 & 0 & +15 & 0 & 0 & +5 & 0 & 0 & +15 & 0 & 0 & 0 & 0 & 0 & 0 \\
$I_{4}$ & 50 & -9 & 0 & 50 & -5 & 0 & 50 & -9 & 0 & $\mathbf{3 5}$ & -5 & +15 & 50 & -10 & 0 \\
$I_{5}$ & 21 & -21 & +25 & $\mathbf{3 1}$ & -25 & +25 & 21 & -21 & +25 & $\mathbf{3 6}$ & -25 & +25 & 36 & -25 & +25 \\
$\mathrm{WP}$ & 126 & +40 & -40 & 126 & +40 & -40 & 126 & +40 & -40 & 126 & +40 & -40 & 121 & +45 & -35 \\
\hline
\end{tabular}

The Stoch and Seq models provide the two extreme solutions in terms of expected cost for all time periods of the scheduling horizon and serve as upper and lower bounds, respectively, for the expected costs of $P-B, V-B$ and $V-B$ gen models. We perform an analogous analysis for the case with a total power load of $344 \mathrm{MW}$ where $\chi^{p}$ gets a positive value and all three improved sequential models achieve the same expected cost with Stoch. This fact illustrates that it is possible in specific 
cases to have an efficient sequential dispatch if the future balancing costs are communicated into the day-ahead market through the operator-defined parameters $\chi$. The additional results are presented in the online appendix (Ordoudis et al., 2019a), along with the development of the operator-defined parameters $\chi$ over the scheduling horizon.

\subsubsection{4-hour Simulation Results}

Additionally, we provide the following results for the whole 24-hour scheduling horizon, where 20 equiprobable wind power scenarios are utilized (available at (Bukhsh, 2017)). Fig. 5 presents the expected cost of the integrated energy system as a function of wind power penetration level, defined as the share of wind power capacity on total system's electricity demand.

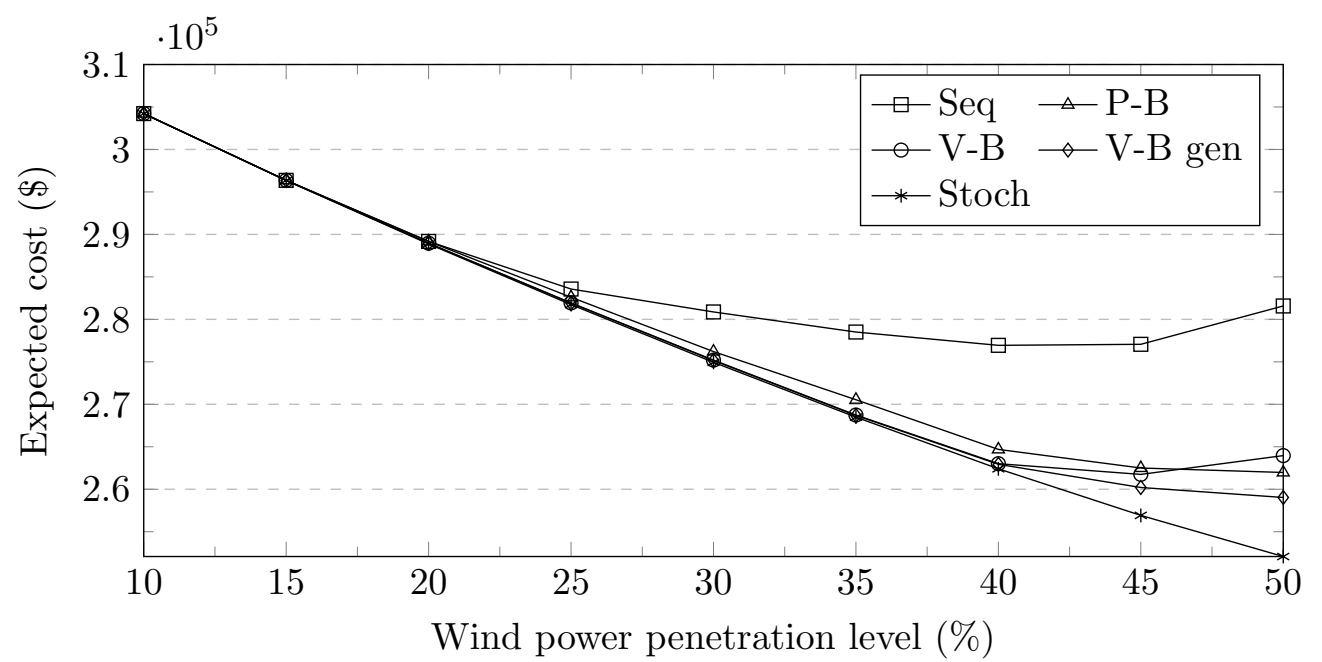

Figure 5: Impact of wind power penetration level on the expected system cost.

The expected cost of Stoch is decreasing with an increase of wind power penetration and achieves the lowest expected cost in all cases. On the other hand, model $S e q$ becomes inefficient for a wind power penetration level above $25 \%$, while even an increase of the expected cost is observed when this level is greater than $40 \%$. The $V-B$ and $V$-B gen models manage to approximate efficiently the solution of Stoch model up to a share of $40 \%$, where they start diverging with an increasing tendency. Note that the expected cost of $V-B$ gen is lower than $V$ - $B$, confirming its higher flexibility to provide an improved day-ahead dispatch. Similarly, $P-B$ attains an expected cost close to the one obtained by Stoch.

We now highlight the main features of the proposed dispatch models in order to provide some additional insights regarding how the scheduling of the power plants is affected at the day-ahead stage. All three $P-B, V-B$ and $V-B$ gen demonstrate a considerable ability to bridge the gap between $S e q$ and Stoch models. They manage to return an expected system cost closer to the stochastic ideal solution, while still dispatch the system based on the merit-order principle and keep the economic properties of Seq. Moreover, they can affect the system dispatch regardless 
of the type of marginal producer, i.e. GFPP or power plant using another fuel. In models $V$ - $B$ and $V$-B gen at least one GFPP would have to be scheduled in order to be able to improve the dispatch of the system, while this restriction does not apply to $P$ - $B$. Additionally, model $V-B$ is able to alter the dispatch of the GFPP with the higher conversion factor. In case the natural gas availability is defined individually for specific areas of the system, the GFPP affected is the one with the greater conversion factor in the specific area. Finally, $V-B$ gen is more flexible than $V-B$ as it can change the dispatch of each individual GFPP. More specifically, the portion of the dayahead power to be produced by GFPPs can be split under different shares in order to reveal more cost-effective regulation in the balancing market. A schematic representation of how the supply curve is redefined under each model is presented in the online appendix (Ordoudis et al., 2019a).

\subsection{Realistic Case-Study}

A more realistic case study is considered to assess the performance of the proposed dispatch model $V-B-N$ when network constraints are included for the real-time operation of the energy system. The integrated energy system consists of the IEEE 24-bus Reliability Test System (RTS) (Grigg et al., 1999) and a 12-node natural gas system based on (He et al., 2017). More specifically, there exist 12 conventional power plants, out of which 4 are GFPPs, 2 wind farms and 3 natural gas suppliers. The offer prices for upward and downward regulation are equal to 1.1 and 0.9 of the day-ahead offer prices. Wind power production is modeled by a set of 25 equiprobable scenarios. The data and network topology are provided in the online appendix, available in (Ordoudis et al., 2019a). Moreover, we introduce a new variant of (9) that defines the natural gas volume availability for specific areas of the integrated energy system, namely $V$ - $B$ area. Two areas are determined in this study including two GFPPs in each one of them. More specifically, GFPP 1 and GFPP 5 are included in area $I$, while GFPP 7 and GFPP 11 in area $I I$. Similarly to Section 4.1, we also examine $V-B-N$ and $V-B-N$ gen. We optimize over a 24-hour scheduling horizon and we set the level of linepack at the end of the day equal to the one at the beginning of the day that is 448,000 kcf.

The expected system cost for different wind power penetration levels is illustrated in Fig. 6 . All models reduce the expected cost compared to $S e q-N$ and this reduction is more significant for higher shares of wind power penetration. Moreover, it can be observed that allowing more degrees of freedom to define natural gas availability allows to capture more efficiently the benefits of Stoch-N.

Additionally, we quantify the benefits of modeling the linepack in the natural gas system by comparing the outcome of the dispatch models when a purely steady-state operation is followed. In this case, the pipelines are not able to store natural gas, hence the inflow and outflow is equal for each time period. Fig. 7 illustrates the relative increase in expected cost when neglecting linepack in comparison with the expected cost presented in Fig. 6. An increase in expected cost is observed for all dispatch models, when neglecting the linepack flexibility. Model Stoch- $N$ is the 


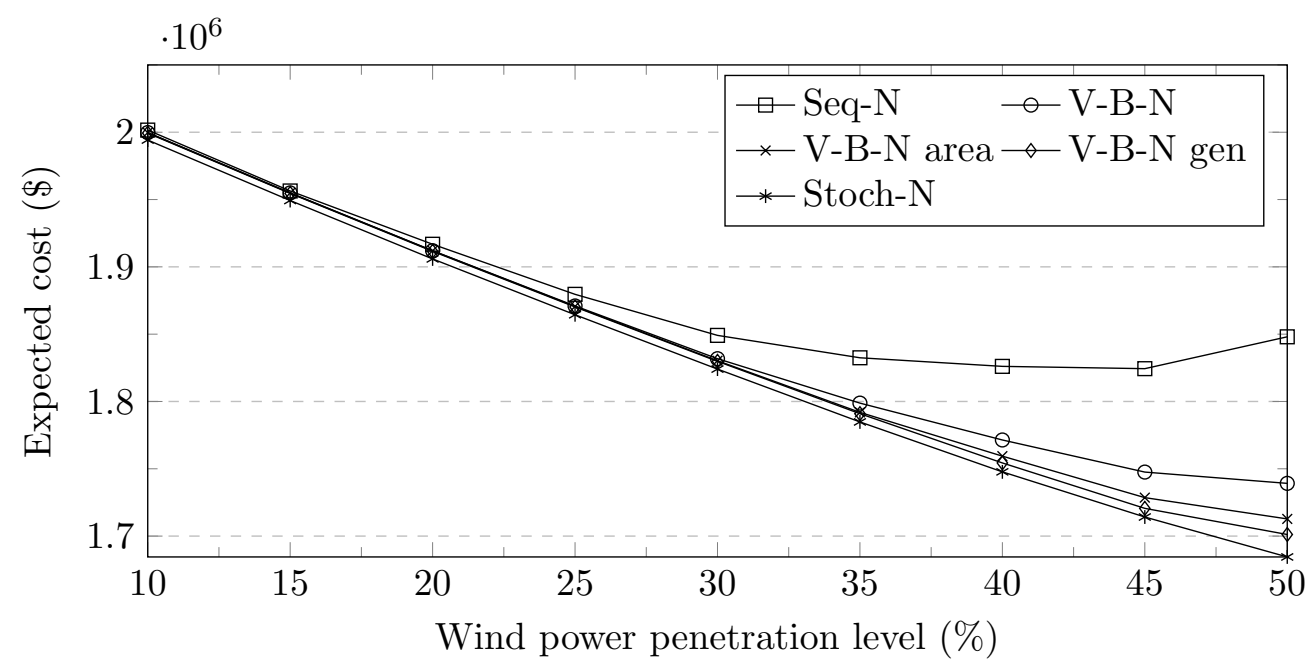

Figure 6: Impact of wind power penetration level on the expected system cost.

most advanced one and accomplishes a consistent decrease of expected cost in both cases, which is only slightly affected by wind power penetration level. On the other hand, $S e q-N$ is the most inefficient and has the greatest increase when linepack is ignored that results in about $3.5 \%$ for a wind power share of 50\%. Regarding the proposed volume-based dispatch models, two trends are noticed. Initially, we observe that the more flexible the procedure is to define natural gas volume availability, the less the outcome is altered up to a $30 \%$ wind power penetration level. Then, the difference in expected cost is higher for $V-B-N$ gen at higher penetration levels. This difference though mainly stems from the efficiency of $V$ - $B$ - $N$ gen to exploit the linepack flexibility and significantly reduce the expected cost in this case, while still having an adequate performance when linepack is neglected. The inclusion of additional storage technologies in the energy system would increase the flexibility and decrease the value of coordination. However, the ranking in expected operational costs among the models is expected to stay the same since there exists an optimal definition of the coordination parameters to be made, which may depend on supply and demand side characteristics of the energy system.

Finally, we illustrate that it is possible for flexible producers to face losses in Stoch- $N$ as cost recovery is only guaranteed in expectation and not for each wind power scenario. On the contrary, models $S e q-N, V-B-N, V-B-N$ area and $V-B-N$ gen respect the merit-order and thus cost recovery is ensured for each scenario. The proposed models reduce the total expected system cost, while protecting the flexible power plants from facing negative profits in any potential realization of uncertainty in the prescribed scenario set. In energy systems with high shares of renewables, these characteristics are highly important in order to efficiently harness existing flexibility and accommodate higher shares of renewables in a cost-effective manner. Table 5 presents the daily profits for the flexible power plant $\mathcal{I}_{3}{ }^{6}$ for wind power penetration level equal to $50 \%$. For Stoch- $N$,

\footnotetext{
${ }^{6}$ The characteristics and location of power plant $\mathcal{I}_{3}$ are shown in the online appendix (Ordoudis et al., 2019a).
} 


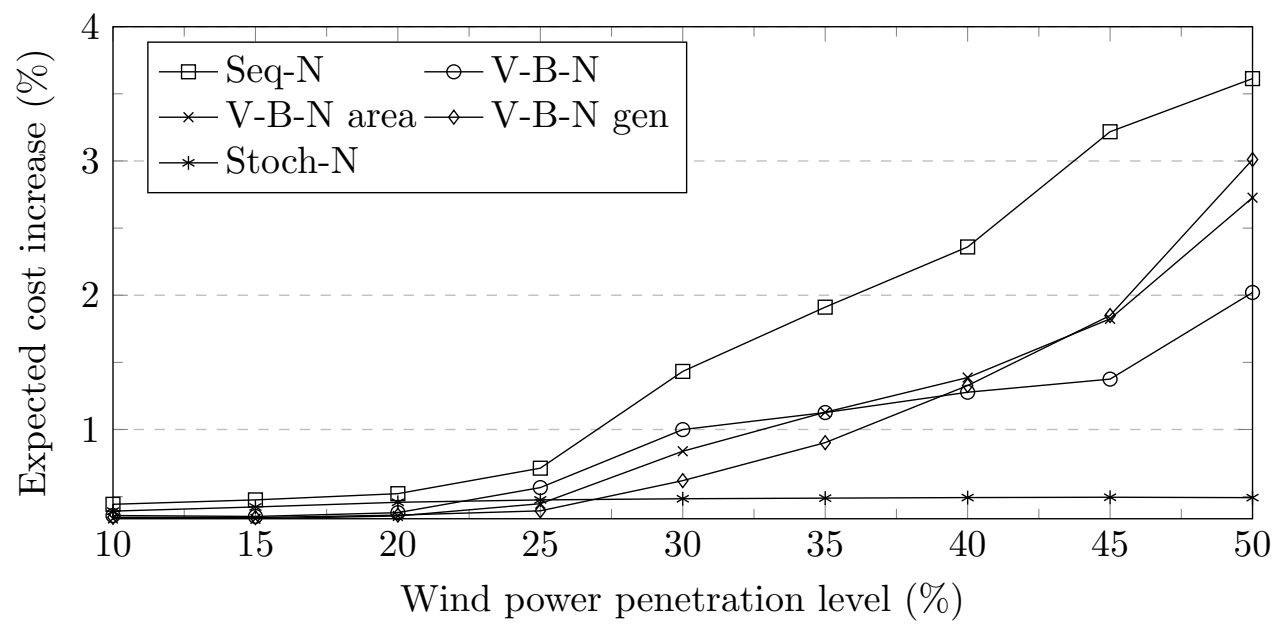

Figure 7: Impact of wind power penetration level on the expected system cost increase when neglecting linepack modeling.

the average losses and the probability of facing a negative profit is shown. Note that the expected profit is significantly higher in $S e q-N$ due to the payments to flexible producers under the very costly balancing actions (e.g. load shedding). Such balancing actions are less often under $V$ - $B$ - $N$, $V-B-N$ area and $V-B-N$ gen; hence, the expected profits are decreased for power plant $\mathcal{I}_{3}$. This is a common observation for all flexible power plants because of the less extreme prices in the balancing market under more efficient dispatch models. An extended discussion regarding this topic is also provided in (Morales et al., 2014).

Table 5: Daily profits of thermal unit $\mathcal{I}_{3}$ in case wind penetration is $50 \%$

\begin{tabular}{cccccc}
\hline & $S e q-N$ & Stoch $-N$ & $V-B-N$ & $V$ - $B$ - $N$ area & $V$ - $B$ - $N$ gen \\
\hline Expected profit (\$) & 239,062 & 4,618 & 73,895 & 52,649 & 47,487 \\
Probability of & 0 & 4 & 0 & 0 & 0 \\
negative profits ${ }^{*}(\%)$ & & & 0 & 0 & 0 \\
Average losses (\$) & 0 & -46.7 & 0 & 0 \\
\hline
\end{tabular}

* Based on the available scenario set $\Omega$.

The optimization problems were solved using CPLEX 12.6.2 under GAMS on a stationary computer with Intel i7 4-core processor clocking at $3.4 \mathrm{GHz}$ and $8 \mathrm{~GB}$ of RAM. The average solution time is presented in Table 6 for both case studies. It can be noticed that $P$ - $B$ has significantly higher solution time. This is due to the greater number of binary variables required for the linearization of complementarity constraints in the KKT conditions, since the balancing market is also included in the lower-level problem of the bilevel formulation. Moreover, the time-coupling constraint that ensures cost neutrality for the system operator at the day-ahead stage also increases the complexity of the problem. 
Table 6: Average solution time in seconds

\begin{tabular}{ccccccc}
\hline Model & $S e q$ & Stoch & $V-B$ & - & $V$ - $B$ gen & $P-B$ \\
\hline Tailored Case-Study & 0.3 & 0.6 & 1.5 & - & 1.5 & 520 \\
\hline Model & $S e q-N$ & Stoch $-N$ & $V-B-N$ & $V$ - $B$ - $N$ area & $V$ - $B$ - $N$ gen & - \\
\hline Realistic Case-Study & 48 & 1,100 & 2,920 & 1,409 & 1,034 & - \\
\hline
\end{tabular}

\section{Conclusion}

This paper proposed a methodology to optimally define the natural gas volume availability for power production in an integrated electricity and natural gas system under high shares of stochastic renewables. Releasing in the day-ahead market a proper amount of natural gas to be consumed by the GFPPs allows us to increase the efficiency of the sequential market design via creating an implicit link between the day-ahead and balancing markets. The current sequential market structures are highly challenged from the increased uncertainty and variability introduced by renewables, since the description of uncertain parameters is performed in a deterministic way. Using the stochastic dispatch model as an ideal benchmark, the proposed volume-based model manages to bridge the efficiency gap between the sequential and stochastic dispatch models. The optimal setting of natural gas availability is achieved through a stochastic bilevel program that anticipates the balancing costs, while its outcome can be directly incorporated in the current market structure. In order to fully exploit the flexibility of the integrated energy system and to enhance the overall system efficiency, we approximate the natural gas system dynamics by modeling linepack that plays an important role in short-term operations. Moreover, a comparison with a price-based model that alters the natural gas price perceived by GFPPs to achieve an implicit temporal link is performed.

Our analysis illustrated that an intelligent adjustment of natural gas volumes or price can significantly improve the efficiency of the sequential dispatch model and bring its expected system cost closer to ideal solution of the stochastic model. The utilization of such decision-support tools facilitates the integration of renewables and captures the benefits of the stochastic dispatch model, while respecting the least-cost merit-order principle and its economic properties. Hence, the operators can directly utilize the outcomes of these decision-support tools or gain insights about the interactions of the electricity and gas systems in order to design new market products and refine the existing market structure. For future work, more detailed models for the electricity and natural gas systems can be considered, as the utilization of AC power flow, the inclusion of unit commitment constraints along with start-up and no-load costs for the thermal power plants and the incorporation of compressors' fuel consumption in the natural gas system. Regarding the modeling of power plants, more details can be taken into account such as quadratic functions to characterize fuel consumption, inter-temporal constraints, quadratic cost functions and varying efficiency across the power production range. Finally, potential computational challenges can be tackled by the use of decomposition techniques. 


\section{Acknowledgment}

The work of Christos Ordoudis and Pierre Pinson is partly funded by the Danish Strategic Research Council (DSF) through CITIES project, No. 1305-00027B/DSF. The work of Jalal Kazempour is supported by the Danish Energy Technology Development and Demonstration Programme (EUDP) through the CORE project (64017-0005). The work of Stefanos Delikaraoglou is funded by the Commission for Technology and Innovation of Switzerland: Project No. 18801.1 PFIW-IW. Finally, we would like to thank Prof. Benjamin F. Hobbs for his feedback on the proposed coordination methodology and for pointing out its relation with the "maximum gas burn" constraint utilized by the California Independent System Operator (CAISO).

\section{Appendix}

Proposition 1. In the sequential clearing of day-ahead and balancing markets, the profits of flexible producers, who can be re-dispatched in real-time operation, over the scheduling horizon are nonnegative (i.e. electricity and natural gas prices guarantee cost recovery). For thermal power plants, that is

$$
\sum_{t \in T}\left[p_{i_{c}, t}\left(\hat{\lambda}_{t}^{E}-C_{i_{c}}\right)+\Delta p_{i_{c}, \omega^{\prime}, t}\left(\tilde{\lambda}_{\omega^{\prime}, t}^{E}-C_{i_{c}}\right)\right] \geq 0
$$

and for GFFPs that is

$$
\sum_{t \in T}\left[p_{i_{g}, t}\left(\hat{\lambda}_{t}^{E}-\phi_{i_{g}} \hat{\lambda}_{t}^{G}\right)+\Delta p_{i_{g}, \omega^{\prime}, t}\left(\tilde{\lambda}_{\omega^{\prime}, t}^{E}-\phi_{i_{g}} \tilde{\lambda}_{\omega^{\prime}, t}^{G}\right)\right] \geq 0 .
$$

Proof of Proposition 1. Notice that Seq model is formulated as two optimization problems in (1) and (2). Considering a different optimization problem for each market participant in $S e q$ model and the market-clearing conditions for each trading floor (i.e. day-ahead and balancing), a twosettlement equilibrium model $S e q-E q$ can be formulated by writing the KKT conditions for each individual optimization model. This set of KKT conditions is identical to those conditions associated with the $S e q$ model, which proves that $S e q$ and $S e q-E q$ are equivalent. Thus, any solution of one model is a solution of the other model too. The aforementioned statement holds if the problems are convex. An extensive discussion on this topic and presentation of the approach to equivalently formulate the equilibrium and optimization models is provided by Kazempour \& Hobbs (2018a) and Kazempour \& Hobbs (2018b). Focusing to the equilibrium model $S e q-E q$, the profit maximization problem of each thermal power producer $i_{c}$ for the day-ahead stage writes as follows,

$$
\begin{aligned}
\left\{\max _{p_{i_{c}, t}}\right. & \sum_{t \in T}\left[p_{i_{c}, t}\left(\hat{\lambda}_{t}^{\mathrm{E}}-C_{i_{c}}\right)\right] \\
\text { s.t. } & \left.0 \leq p_{i_{c}, t} \leq P_{i}^{\mathrm{max}}: \underline{\mu}_{i_{c}, t}^{\mathrm{P}}, \bar{\mu}_{i_{c}, t}^{\mathrm{P}}\right\}, \quad \forall i_{c}, t .
\end{aligned}
$$


Since program (13) is linear and thus convex, the strong duality theorem holds for the optimal solution and

$$
\sum_{t \in T}\left[p_{i_{c}, t}\left(\hat{\lambda}_{t}^{\mathrm{E}}-C_{i_{c}}\right)\right]=\sum_{t \in T} \bar{\mu}_{i_{c}, t}^{\mathrm{P}} P_{i_{c}}^{\max }
$$

where $\bar{\mu}_{i_{c}, t}^{\mathrm{P}} \geq 0$ and $P_{i_{c}}^{\max } \geq 0$ which shows that the profits in the day-ahead market are nonnegative. Similarly, the profit maximization problem for the balancing market can be written by having the day-ahead decision $p_{i_{c}, t}^{*}$ fixed,

$$
\begin{aligned}
\left\{\max _{\Delta p_{i, \omega^{\prime}, t}}\right. & \sum_{t \in T}\left[\Delta p_{i, \omega^{\prime}, t}\left(\tilde{\lambda}_{\omega^{\prime}, t}^{\mathrm{E}}-C_{i_{c}}\right)\right] \\
\text { s.t. } & -p_{i_{c}, t}^{*} \leq \Delta p_{i_{c}, \omega^{\prime}, t} \leq P_{i_{c}}^{\max }-p_{i_{c}, t}^{*}: \underline{\mu}_{i_{c}, \omega^{\prime}, t}^{\mathrm{R}}, \bar{\mu}_{i_{c}, \omega^{\prime}, t}^{\mathrm{R}}, \\
& \left.-P_{i}^{-} \leq \Delta p_{i_{g}, \omega^{\prime}, t} \leq P_{i}^{+}: \underline{\mu}_{i_{c}, \omega^{\prime}, t}^{\mathrm{RR}}, \bar{\mu}_{i_{c}, \omega^{\prime}, t}^{\mathrm{RR}}\right\}, \quad \forall i_{c}, \omega^{\prime}, t .
\end{aligned}
$$

Furthermore, strong duality theorem for the optimal solution of program (15),

$$
\sum_{t \in T}\left[\Delta p_{i_{c}, \omega^{\prime}, t}\left(\tilde{\lambda}_{\omega^{\prime}, t}^{\mathrm{E}}-C_{i_{c}}\right)\right]=\sum_{t \in T}\left(\bar{\mu}_{i_{c}, \omega^{\prime}, t}^{\mathrm{R}}\left(P_{i_{c}}^{\mathrm{max}}-p_{i_{c}, t}^{*}\right)+\underline{\mu}_{i_{c}, \omega^{\prime}, t}^{\mathrm{R}} p_{i_{c}, t}^{*}+\bar{\mu}_{i_{c}, \omega^{\prime}, t}^{\mathrm{RR}} P_{i_{c}}^{+}+\underline{\mu}_{i_{c}, \omega^{\prime}, t}^{\mathrm{RR}} P_{i_{c}}^{-}\right)
$$

where $\bar{\mu}_{i_{c}, \omega^{\prime}, t}^{\mathrm{R}}, \underline{\mu}_{i_{c}, \omega^{\prime}, t}^{\mathrm{R}}, \bar{\mu}_{i_{c}, \omega^{\prime}, t}^{\mathrm{RR}}, \underline{\mu}_{i_{c}, \omega^{\prime}, t}^{\mathrm{RR}} \geq 0$. Moreover, the quantities $\left(P_{i_{c}}^{\max }-p_{i_{c}, t}^{*}\right), p_{i_{c}, t}^{*}, P_{i_{c}}^{+}, P_{i_{c}}^{-} \geq 0$. Thus, the profits in balancing market are also non-negative. Since cost recovery holds for $S e q-E q$ model, then it means that it is also ensured in optimization model $S e q$ due to their equivalence. A similar proof can be written for the GFPPs, where the marginal cost $C_{i_{c}}$ is replaced by $\phi_{i_{g}} \hat{\lambda}_{t}^{\mathrm{G}}$ and $\phi_{i_{g}} \tilde{\lambda}_{\omega^{\prime}, t}^{\mathrm{G}}$ for the day-ahead and balancing markets, respectively. The claim then follows.

The analogous proof regarding cost recovery for flexible producers in the stochastic dispatch model Stoch is presented by Morales et al. (2012). In this case, cost recovery holds only in expectation and not per scenario realization of stochastic power production. Morales et al. (2014) provide a detailed discussion on the topic. Finally, a stochastic market-clearing model that ensures cost recovery and revenue adequacy per scenario is proposed by Kazempour et al. (2018).

\section{Nomenclature}

\section{Parameters}

$\Gamma_{z} \quad$ Compressor factor located at natural gas network branch $z[-]$.

$\bar{W}_{j} \quad$ Capacity of stochastic power plant $j[\mathrm{MW}]$.

$\phi_{i_{g}} \quad$ Power conversion factor of natural gas-fired power plant $i_{g}[\mathrm{kcf} / \mathrm{MWh}]$.

$\pi_{\omega} \quad$ Probability of scenario $\omega[-]$.

$\tilde{M}_{m, u}$ Sufficiently large constant for pipeline $(\mathrm{m}, \mathrm{u})$ [-]. 
$\widehat{W}_{j, t} \quad$ Expected power production by stochastic power plant $j$ in period $t$ [MW].

$B_{n, r} \quad$ Absolute value of the susceptance of line (n,r) [per unit].

$C_{i}^{+}, C_{i}^{-} \mathrm{Up} /$ down regulation offer price of dispatchable power plant $i$ [\$/MWh].

$C_{k}^{+}, C_{k}^{-} \mathrm{Up} /$ down regulation offer price of natural gas producer $k[\$ / \mathrm{kcf}]$.

$C^{\text {sh,E }}$ Cost of electricity load shedding [\$/MWh].

$C^{\text {sh, G }}$ Cost of natural gas load shedding $[\$ / \mathrm{kcf}]$.

$C_{i} \quad$ Day-ahead offer price of dispatchable power plant $i[\$ / \mathrm{MWh}]$.

$C_{k} \quad$ Day-ahead offer price of natural gas producer $k[\$ / \mathrm{kcf}]$.

$D_{r_{e}, t}^{\mathrm{E}} \quad$ Electricity demand $r_{e}$ and in period $t[\mathrm{MW}]$.

$D_{r_{g}, t}^{\mathrm{G}} \quad$ Natural gas demand $r_{g}$ and in period $t[\mathrm{kcf} / \mathrm{h}]$.

$F_{z}^{\mathrm{A}} \quad$ Daily contract limit of natural gas pipeline $z$ [kcf].

$F_{\psi, t}^{\max }$ Maximum natural gas availability for a specific area $\psi$ containing the group of GFPPs $[\mathrm{kcf} / \mathrm{h}]$.

$F_{n, r}^{\max } \quad$ Transmission capacity of line (n,r) [MW].

$F_{z, t}^{\mathrm{M}} \quad$ Capacity of natural gas pipeline $z$ in period $t[\mathrm{kcf} / \mathrm{h}]$.

$G_{k}^{+}, G_{k}^{-}$Maximum up/down reserve offered by natural gas producer $k[\mathrm{kcf} / \mathrm{h}]$.

$G_{k}^{\max }$ Capacity of natural gas producer $k[\mathrm{kcf} / \mathrm{h}]$.

$K_{m, u}^{\mathrm{f}}$ Natural gas flow (f) constant of pipeline $(\mathrm{m}, \mathrm{u})[\mathrm{kcf} /(\mathrm{psig} \cdot \mathrm{h})]$.

$K_{m, u}^{\mathrm{h}}$ Linepack constant of pipeline $(\mathrm{m}, \mathrm{u})$ [kcf/psig].

$P_{i}^{+}, P_{i}^{-}$Maximum up/down reserve offered by dispatchable power plant $i[\mathrm{MW}]$.

$P_{i}^{\max }$ Capacity of dispatchable power plant $i[\mathrm{MW}]$.

$P R_{m}^{\min } / \max$ Minimum and maximum pressure at node $m[\mathrm{psig}]$.

$W_{j, \omega, t}$ Power production by stochastic power plant $j$ in scenario $\omega$, period $t$ [MW].

$X \quad$ Limit of natural gas price adjustment $[\$ / \mathrm{kcf}]$.

\section{Sets}

$\Omega \quad$ Set of stochastic power production scenarios $\omega$.

$\Psi \quad$ Set of groups of natural gas-fired power plants located in a specific area $\psi$.

$\Theta \quad$ Set of primal optimization variables defined for each optimization model.

$A_{\psi}^{I_{g}} \quad$ Set of natural gas-fired power plants $i_{g}$ located in a specific area $\psi$.

$A_{m}^{I_{g}} \quad$ Set of natural gas-fired power plants $i_{g}$ located at natural gas network node $m$. 
$A_{m}^{K} \quad$ Set of natural gas producers $k$ located at natural gas network node $m$.

$A_{m}^{R_{g}} \quad$ Set of natural gas demands $r_{g}$ located at natural gas network node $m$.

$A_{n}^{I} \quad$ Set of dispatchable power plants $i$ located at electricity network node $n$.

$A_{n}^{J} \quad$ Set of stochastic power plants $j$ located at electricity network node $n$.

$A_{n}^{R_{e}} \quad$ Set of electricity demands $r_{e}$ located at electricity network node $n$.

$I \quad$ Set of dispatchable power plants $i$.

$I_{c} \quad$ Set of thermal power plants $i_{c}\left(I_{c} \subset I\right)$.

$I_{g} \quad$ Set of natural gas-fired power plants $i_{g}\left(I_{g} \subset I\right)$.

$J \quad$ Set of stochastic power plants $j$.

$K \quad$ Set of natural gas producers $k$.

$L \quad$ Set of electricity transmission lines $l$.

$M \quad$ Set of natural gas network nodes $m$.

$N \quad$ Set of electricity network nodes $n$.

$R_{e} \quad$ Set of electricity demands $r_{e}$.

$R_{g} \quad$ Set of natural gas demands $r_{g}$.

$T \quad$ Set of time periods $t$.

$V \quad$ Set of fixed pressure points $v$ for the linearization of Weymouth equation.

$Z \quad$ Set of natural gas network branches $z$.

\section{Variables}

$\chi_{t}^{p} \quad$ Natural gas price adjustment in period $t[\$ / \mathrm{kcf}]$.

$\chi_{\psi, t}^{v} \quad$ Hourly natural gas volume availability for GFPPs' group in specific area $\psi$ in period $t$ [kcf].

$\hat{\lambda}_{t}^{\mathrm{E}} \quad$ Electricity locational marginal price in day-ahead market at period $t[\$ / \mathrm{MWh}]$.

$\hat{\lambda}_{t}^{\mathrm{G}} \quad$ Natural gas locational marginal price in day-ahead market at period $t[\$ / \mathrm{kcf}]$.

$\bar{\chi}_{\psi}^{v} \quad$ Daily natural gas volume availability for GFPPs' group in specific area $\psi[\mathrm{kcf}]$.

$\tilde{\delta}_{n, \omega, t} \quad$ Voltage angle at node $n$ in scenario $\omega$, period $t$ [rad].

$\tilde{\lambda}_{n, \omega, t}^{\mathrm{E}} \quad$ Electricity locational marginal price in balancing market at node $n$ in scenario $\omega$, period $t$ [\$/MWh].

$\tilde{\lambda}_{m, \omega, t}^{\mathrm{G}}$ Natural gas locational marginal price in balancing market at node $m$ in scenario $\omega$, period $t[\$ / \mathrm{kcf}]$.

$g_{k, \omega, t}^{+/-} \quad \mathrm{Up} /$ down regulation provided by natural gas producer $k$ in scenario $\omega$, period $t[\mathrm{kcf} / \mathrm{h}]$.

$g_{k, t} \quad$ Day-ahead dispatch of natural gas producer $k$ in period $t[\mathrm{kcf} / \mathrm{h}]$. 
$h_{m, u, \omega, t}^{\mathrm{r}}$ Average mass of natural gas (linepack) in pipeline (m,u), scenario $\omega$, period $t$ [kcf].

$l_{n / m, \omega, t}^{\mathrm{sh}, \mathrm{E} / \mathrm{G}}$ Electric power and natural gas load shedding at node $n / m$ in scenario $\omega$, period $t[\mathrm{MW}$, $\mathrm{kcf} / \mathrm{h}]$.

$p_{i, \omega, t}^{+/-} \quad \mathrm{Up} /$ down regulation provided by dispatchable power plant $i$ in scenario $\omega$, period $t$ [MW].

$p_{i, t} \quad$ Day-ahead dispatch of power plants $i$ in period $t[\mathrm{MW}]$.

$p r_{m, \omega, t}^{\mathrm{r}}$ Pressure at node $m$ in scenario $\omega$, period $t$ [psig].

$\underset{m, u, \omega, t}{\text { in/out,r }}$ In- and outflow natural gas rates of pipeline $(\mathrm{m}, \mathrm{u})$ in scenario $\omega$, period $t[\mathrm{kcf} / \mathrm{h}]$.

$q_{m, u} \quad$ Natural gas flow in pipeline $(\mathrm{m}, \mathrm{u})[\mathrm{kcf} / \mathrm{h}]$.

$w_{j, \omega, t}^{\mathrm{sp}}$ Power spilled by stochastic power plant $j$ in scenario $\omega$, period $t$ [MW].

$w_{j, t} \quad$ Day-ahead dispatch of wind power plants $j$ in period $t[\mathrm{MW}]$.

$y_{m, u, \omega, t}^{\mathrm{r}}$ Binary variable defining the direction of the natural gas flow in pipeline $(\mathrm{m}, \mathrm{u})$, scenario $\omega$, period $t\{0,1\}$.

\section{References}

Alabdulwahab, A., Abusorrah, A., Zhang, X., \& Shahidehpour, M. (2015). Coordination of interdependent natural gas and electricity infrastructures for firming the variability of wind energy in stochastic day-ahead scheduling. IEEE Transactions on Sustainable Energy, 6, 606-615.

Borraz-Sanchez, C., Bent, R., Backhaus, S., Hijazi, H., \& Van Hentenryck, P. (2016). Convex relaxations for gas expansion planning. INFORMS Journal on Computing, 28, 645-656.

Bukhsh, W. (2017). Data for stochastic multiperiod optimal power flow problem. https://sites.google.com/site/datasmopf/ Accessed 20.11.18.

CAISO (2017). Aliso Canyon gas-electric coordination. http://www.caiso.com/Documents/DraftFinalProposalAlisoCanyonGas-ElectricCoordinationPhase3.pdf Accessed 20.11.18.

Correa-Posada, C. M., \& Sanchez-Martin, P. (2014). Integrated power and natural gas model for energy adequacy in short-term operation. IEEE Transactions on Power Systems, 30, 3347-3355.

Delikaraoglou, S., \& Pinson, P. (2019). Optimal allocation of HVDC interconnections for exchange of energy and reserve capacity services. Energy Systems, 10, 635-675.

Dvorkin, V., Delikaraoglou, S., \& Morales, J. M. (2019). Setting reserve requirements to approximate the efficiency of the stochastic dispatch. IEEE Transactions on Power Systems, 34, 1524-1536.

Fodstad, M., Midthun, K. T., \& Tomasgard, A. (2015). Adding flexibility in a natural gas transportation network using interruptible transportation services. European Journal of Operational Research, 243, 647-657.

Gil, J., Caballero, A., \& Conejo, A. J. (2014). Power cycling: Ccgts: The critical link between the electricity and natural gas markets. IEEE Power and Energy Magazine, 12, 40-48.

Gribik, P., Hogan, W., \& Pope, S. (2007). Market-clearing prices and energy uplift. Online: https://sites.hks.harvard.edu/fs/whogan/Gribik_Hogan_Pope_Price_Uplift_123107.pdf.

Grigg, C., Wong, P., Albrecht, P., Allan, R., Bhavaraju, M., Billinton, R., Chen, Q., Fong, C., Haddad, S., Kuruganty, S., Li, W., Mukerji, R., Patton, D., Rau, N., Reppen, D., Schneider, A., Shahidehpour, M., \& Singh, C. (1999). The IEEE reliability test system-1996. A report prepared by the reliability test system task force of the application of probability methods subcommittee. IEEE Transactions on Power Systems, 14, 1010-1020. 
He, C., Wu, L., Liu, T., \& Shahidehpour, M. (2017). Robust co-optimization scheduling of electricity and natural gas systems via ADMM. IEEE Transactions on Sustainable Energy, 8, 658-670.

Henriot, A., \& Glachant, J.-M. (2014). Capacity remuneration mechanisms in the European market: Now but how? European University Institute (EUI), Robert Schuman Centre of Advanced Studies (RSCAS), .

Hibbard, P. J., \& Schatzki, T. (2012). The interdependence of electricity and natural gas: Current factors and future prospects. The Electricity Journal, 25, 6-17.

Jensen, T. V., Kazempour, J., \& Pinson, P. (2018). Cost-optimal ATCs in zonal electricity markets. IEEE Transactions on Power Systems, 33, 3624-3633.

Kazempour, J., \& Hobbs, B. F. (2018a). Value of flexible resources, virtual bidding, and self-scheduling in twosettlement electricity markets with wind generation - part I: Principles and competitive model. IEEE Transactions on Power Systems, 33, 749-759.

Kazempour, J., \& Hobbs, B. F. (2018b). Value of flexible resources, virtual bidding, and self-scheduling in twosettlement electricity markets with wind generation - part II: ISO models and application. IEEE Transactions on Power Systems, 33, 760-770.

Kazempour, J., Pinson, P., \& Hobbs, B. F. (2018). A stochastic market design with revenue adequacy and cost recovery by scenario: Benefits and costs. IEEE Transactions on Power Systems, 33, 3531-3545.

Meibom, P., Hilger, K. B., Madsen, H., \& Vinther, D. (2013). Energy comes together in denmark: The key to a future fossil-free danish power system. IEEE Power and Energy Magazine, 11, 46-55.

Misra, S., Fisher, M. W., Backhaus, S., Bent, R., Chertkov, M., \& Pan, F. (2015). Optimal compression in natural gas networks: A geometric programming approach. IEEE Transactions on Control of Network Systems, $2,47-56$.

Morales, J. M., Conejo, A. J., Liu, K., \& Zhong, J. (2012). Pricing electricity in pools with wind producers. IEEE Transactions on Power Systems, 27, 1366-1376.

Morales, J. M., Zugno, M., Pineda, S., \& Pinson, P. (2014). Electricity market clearing with improved scheduling of stochastic production. European Journal of Operational Research, 235, 765-774.

O’Neill, R. P., Sotkiewicz, P. M., Hobbs, B. F., Rothkopf, M. H., \& Stewart, W. R. (2005). Efficient market-clearing prices in markets with nonconvexities. European Journal of Operational Research, 164, $269-285$.

Ordoudis, C., Delikaraoglou, S., Kazempour, J., \& Pinson, P. (2019a). Electronic companion - Market-based coordination of integrated electricity and natural gas systems under uncertain supply. https://zenodo.org/record/3596909 Accessed: 02.01.2020.

Ordoudis, C., Delikaraoglou, S., Pinson, P., \& Kazempour, J. (2017). Exploiting flexibility in coupled electricity and natural gas markets: A price-based approach. In 2017 IEEE Manchester PowerTech (pp. 1-6).

Ordoudis, C., Pinson, P., \& Morales, J. M. (2019b). An integrated market for electricity and natural gas systems with stochastic power producers. European Journal of Operational Research, 272, 642 - 654 .

Pinson, P., Mitridati, L., Ordoudis, C., \& Østergaard, J. (2017). Towards fully renewable energy systems : Experience and trends in Denmark. CSEE Journal of Power and Energy Systems, 3, 26-35.

Pozo, D., Sauma, E., \& Contreras, J. (2017). Basic theoretical foundations and insights on bilevel models and their applications to power systems. Annals of Operations Research, 254, 303-334.

Pritchard, G., Zakeri, G., \& Philpott, A. (2010). A single-settlement, energy-only electric power market for unpredictable and intermittent participants. Operations Research, 58, 1210-1219.

Rømo, F., Tomasgard, A., Hellemo, L., Fodstad, M., Eidesen, B. H., \& Pedersen, B. (2009). Optimizing the Norwegian natural gas production and transport. Interfaces (Providence), 39, 46-56.

Schwele, A., Ordoudis, C., Kazempour, J., \& Pinson, P. (2019). Coordination of power and natural gas systems: Convexification approaches for linepack modeling. In 2019 IEEE Milan PowerTech (pp. 1-6).

Tomasgard, A., Rømo, F., Fodstad, M., \& Midthun, K. (2007). Optimization models for the natural gas value chain. In G. Hasle, K.-A. Lie, \& E. Quak (Eds.), Geometric Modelling, Numerical Simulation, and Optimization: Applied Mathematics at SINTEF (pp. 521-558). Berlin, Heidelberg: Springer Berlin Heidelberg. 
U.S. Energy Information Administration (2016). International Energy Outlook 2016. Technical Report May 2016 U.S. Energy Information Administration.

Von Stackelberg, H. (2011). Market Structure and Equilibrium. Springer Berlin Heidelberg.

Wang, C., Wei, W., Wang, J., Wu, L., \& Liang, Y. (2018). Equilibrium of interdependent gas and electricity markets with marginal price based bilateral energy trading. IEEE Transactions on Power Systems, 33, 4854-4867.

Zavala, V. M., Kim, K., Anitescu, M., \& Birge, J. (2017). A stochastic electricity market clearing formulation with consistent pricing properties. Operations Research, 65, 557-576.

Zeinalzadeh, A., Aguiar, N., Baros, S., Annaswamy, A. M., Chakraborty, I., \& Gupta, V. (2017). Using natural gas reserves to mitigate intermittence of renewables in the day ahead market. In 56th IEEE Annual Conference on Decision and Control (CDC) (pp. 3896-3901).

Zhao, B., Conejo, A. J., \& Sioshansi, R. (2016). Unit commitment under gas-supply uncertainty and gas-price variability. IEEE Transactions on Power Systems, 32, 2394-2405.

Zlotnik, A., Chertkov, M., \& Backhaus, S. (2015). Optimal control of transient flow in natural gas networks. In 54 th IEEE Conference on Decision and Control (CDC) (pp. 4563-4570).

Zlotnik, A., Roald, L., Backhaus, S., Chertkov, M., \& Andersson, G. (2017). Coordinated scheduling for interdependent electric power and natural gas infrastructures. IEEE Transactions on Power Systems, 32, 600-610. 